\title{
GEOMETRIC INVARIANTS OF SPECTRUM OF THE NAVIER-LAMÉ OPERATOR
}

\author{
GENQIAN LIU \\ School of Mathematics and Statistics, Beijing Institute of Technology, Beijing 100081, China \\ Emails: liugqz@bit.edu.cn
}

\begin{abstract}
For a compact connected Riemannian $n$-manifold $(\Omega, g)$ with smooth boundary, we explicitly calculate the first two coefficients $a_{0}$ and $a_{1}$ of the asymptotic expansion of $\sum_{k=1}^{\infty} e^{-t \tau_{k}^{\mp}}=a_{0} t^{-n / 2} \mp$ $a_{1} t^{-(n-1) / 2}+a_{2}^{\mp} t^{-(n-2) / 2}+\cdots+a_{m}^{\mp} t^{-(n-m) / 2}+O\left(t^{-(n-m-1) / 2}\right)$ as $t \rightarrow 0^{+}$, where $\tau_{k}^{-}$(respectively, $\tau_{k}^{+}$) is the $k$-th Navier-Lamé eigenvalue on $\Omega$ with Dirichlet (respectively, Neumann) boundary condition. These two coefficients provide precise information for the volume of the elastic body $\Omega$ and the surface area of the boundary $\partial \Omega$ in terms of the spectrum of the Navier-Lamé operator. This gives an answer to an interesting and open problem mentioned by Avramidi in [10. More importantly, our method is valid to explicitly calculate all the coefficients $a_{l}^{\mp}, 2 \leq l \leq m$, in the above asymptotic expansion. As an application, we show that an $n$-dimensional ball is uniquely determined by its Navier-Lamé spectrum among all bounded elastic bodies with smooth boundary.
\end{abstract}

\section{INTRODUCTION}

For the Navier-Lamé elastic wave equations, one of the most important problems is to study the shape of the elastic body from its vibrational frequencies, because this kind of geometric property reveals the essential behavior of the elastic body.

Let $(\Omega, g)$ be a Riemannian $n$-manifold with smooth boundary $\partial \Omega$. Let $P_{g}$ be the Navier-Lamé operator:

$$
P_{g} \mathbf{u}:=\mu \nabla^{*} \nabla \mathbf{u}-(\mu+\lambda) \operatorname{grad} \operatorname{div} \mathbf{u}-\mu \operatorname{Ric}(\mathbf{u}), \quad \mathbf{u}=\left(u^{1}, \cdots, u^{n}\right),
$$

where $\mu$ and $\lambda$ are Lamé parameters satisfying $\mu>0$ and $\mu+\lambda \geq 0, \nabla^{*} \nabla$ is the Bochner Laplacian (see (2.11) in section 2, or (2.12) of [56]), div and grad are the usual divergence and gradient operators, and

$$
\operatorname{Ric}(\mathbf{u})=\left(\sum_{k, l=1}^{n} R_{l k}^{k} 1 u^{l}, \sum_{k, l=1}^{n} R_{l k}^{k} u^{l}, \cdots, \sum_{k, l=1}^{n} R_{l k}^{k} u^{l}\right)
$$

denotes the action of Ricci tensor $\mathrm{R}_{l}^{j}:=\sum_{k=1}^{n} R_{l k}^{k j}$ on $\mathbf{u}$.

The natural boundary conditions for $P_{g}$ (see, Lemma 2.1.1 in [56]) include prescribing $\left.\mathbf{u}\right|_{\partial \Omega}$, Dirichlet type, and

$$
\frac{\partial \mathbf{u}}{\partial \nu}:=2 \mu(\operatorname{Def} \mathbf{u})^{\#} \nu+\lambda(\operatorname{div} \mathbf{u}) \nu \quad \text { on } \partial \Omega,
$$

Neumann type, where (Def $\mathbf{u})_{j k}=\frac{1}{2}\left(u_{j ; k}+u_{k ; j}\right)$ is the strain tensor, \# is the sharp operator (for a tensor) by raising index and $\nu$ is the unit inner normal to $\partial \Omega$. We denote by $P_{g}^{-}$and $P_{g}^{+}$the Navier-Lamé operators with the Dirichlet and Neumann boundary conditions, respectively. Since $P_{g}^{-}$(respectively, $P_{g}^{+}$) is an unbounded, self-adjoint and positive (respectively, nonnegative) operator in $\left[H_{0}^{1}(\Omega)\right]^{n}$ (respectively, $\left[H^{1}(\Omega)\right]^{n}$ ) with discrete spectrum $0<\tau_{1}^{-}<\tau_{2}^{-} \leq \cdots \leq \tau_{k}^{-} \leq \cdots \rightarrow+\infty$ (respectively, $0 \leq \tau_{1}^{+}<\tau_{2}^{+} \leq \cdots \leq \tau_{k}^{+} \leq \cdots \rightarrow+\infty$ ), one has (see [10, [66], [1], [56], 47] or [35])

$$
P_{g}^{\mp} \mathbf{u}_{k}^{\mp}=\tau_{k}^{\mp} \mathbf{u}_{k}^{\mp},
$$

MSC 2020: 74B05, 35K50, 35P20, 35S05.

Key Words: Navier-Lamé eigenvalues; Pseudodifferential operators; Navier-Lamé semigroup; Asymptotic expansion. 
where $\mathbf{u}_{k}^{-} \in\left[H_{0}^{1}(\Omega)\right]^{n}$ (respectively, $\mathbf{u}_{k}^{+} \in\left[H^{1}(\Omega)\right]^{n}$ ) is the eigenvector corresponding to eigenvalue $\tau_{k}^{-}$ (respectively, $\tau_{k}^{+}$). (1.4) can be rewritten as

$$
\begin{cases}\mu \nabla^{*} \nabla \mathbf{u}_{k}^{-}-(\mu+\lambda) \operatorname{grad} \operatorname{div} \mathbf{u}_{k}^{-}-\mu \operatorname{Ric}\left(\mathbf{u}_{k}^{-}\right)=\tau_{k}^{-} \mathbf{u}_{k}^{-} & \text {in } \Omega, \\ \mathbf{u}_{k}^{-}=0 & \text { on } \partial \Omega\end{cases}
$$

and

$$
\begin{cases}\mu \nabla^{*} \nabla \mathbf{u}_{k}^{+}-(\mu+\lambda) \operatorname{grad} \operatorname{div} \mathbf{u}_{k}^{+}-\mu \operatorname{Ric}\left(\mathbf{u}_{k}^{+}\right)=\tau_{k}^{+} \mathbf{u}_{k}^{+} & \text {in } \Omega, \\ \frac{\partial \mathbf{u}_{k}^{+}}{\partial \nu}=0 & \text { on } \partial \Omega .\end{cases}
$$

Clearly, the eigenvalue problems (1.5) and (1.6) can be immediately obtained by considering the solutions of the form $\mathbf{v}(t, x)=T(t) \mathbf{u}(x)$ in the following Navier-Lamé elastodynamic wave equations:

$$
\begin{cases}\frac{\partial^{2} \mathbf{v}^{-}}{\partial t^{2}}+\mu \nabla^{*} \nabla \mathbf{v}^{-}-(\mu+\lambda) \operatorname{grad} \operatorname{div} \mathbf{v}^{-}-\mu \operatorname{Ric}\left(\mathbf{v}^{-}\right)=0 & \text { in }(0,+\infty) \times \Omega, \\ \mathbf{v}^{-}=0 & \text { on }(0,+\infty) \times \partial \Omega \\ \mathbf{v}^{-}(0, x)=\mathbf{v}_{0} \quad \frac{\partial \mathbf{v}^{-}}{\partial t}(0, x)=0 & \text { on }\{0\} \times \Omega\end{cases}
$$

and

$$
\begin{cases}\frac{\partial^{2} \mathbf{v}^{+}}{\partial \mathbf{t}^{2}}+\mu \nabla^{*} \nabla \mathbf{v}^{+}-(\mu+\lambda) \operatorname{grad} \operatorname{div} \mathbf{v}^{+}-\mu \operatorname{Ric}\left(\mathbf{v}^{+}\right)=0 & \text { in }(0,+\infty) \times \Omega, \\ \frac{\partial \mathbf{v}^{+}}{\partial \nu}=0 & \text { on }(0,+\infty) \times \partial \Omega, \\ \mathbf{v}^{+}(0, x)=\mathbf{v}_{0} \quad \frac{\partial \mathbf{v}^{+}}{\partial t}(0, x)=0 & \text { on }\{0\} \times \Omega .\end{cases}
$$

In three spatial dimensions, the elastic wave equations (1.7) and (1.8) describe the propagations of waves in an isotropic homogeneous elastic medium. The elasticity of the material provides the restoring force of the wave. Most solid materials are elastic, so these two equations describe such phenomena as seismic waves in the Earth, ultrasonic waves used to detect flaws in materials and the deformations of thin elastic shells whose middle surface must stay inside a given surface in the three-dimensional Euclidean space. Equivalently, we can also rewrite the equation in (1.7) (or (1.8)) into another form (cf. [56] and [57), as it must account for both longitudinal and transverse motion (in three spatial dimensions):

$$
\frac{\partial^{2} \mathbf{v}^{\mp}}{\partial t^{2}}+\mu \operatorname{curl} \operatorname{curl} \mathbf{v}^{\mp}-(2 \mu+\lambda) \operatorname{grad} \operatorname{div} \mathbf{v}^{\mp}-2 \mu \operatorname{Ric}\left(\mathbf{v}^{\mp}\right)=0 .
$$

In particular, if $\operatorname{div} \mathbf{v}^{\mp}$ are set to zero, (1.9) becomes (effectively) Maxwell's equations for the propagation of the electric field $\mathbf{v}^{\mp}$, which has only transverse waves (see [31, [19, 73]). In addition, if $\lambda+\mu=0$, then (1.7) and (1.8) reduce to the classical wave equations, which has only longitudinal waves. For the derivation of the Navier-Lamé elastic wave equations, its mechanical meaning and the explanation of the Dirichlet and Neumann boundary conditions, we refer the reader to [56] for the case of Riemannian manifold and to [12, [23, 34, 39, 46], 40, 70, for the case of Euclidean space.

The Navier-Lamé eigenvalues are physical quantities because they just are the square of vibrational frequencies of an elastic body in two or three dimensions. And these basic physical quantities can be measured experimentally. An interesting question, which is similar to the famous Kac question for the DirichletLaplacian (see [38, [59] or [77] ), is: "can one hear the shape of an elastic body by hearing the vibrational frequencies (or pitches) of the elastic body?" More precisely, does the spectrum of the Navier-Lamé operator determine the geometry of an elastic body (see [10])?

In the special case of $\mu+\lambda=0$ (i.e., the Navier-Lamé operator reduces to the Laplace operator), a celebrated result of the spectral (geometric) invariants had been obtained by McKean and Singer 60. They proved the famous Kac conjecture and gave an explicit expression to the first three coefficients of asymptotic expansion for the heat trace of the Laplacian on a bounded domain $\Omega$ of a Riemannian manifold:

$$
\begin{aligned}
\sum_{k=1}^{\infty} e^{-\beta_{k}^{\mp} t}= & (4 \pi t)^{-n / 2}\left(\operatorname{Vol}(\Omega) \mp \frac{1}{4} \sqrt{4 \pi t} \operatorname{Vol}(\partial \Omega)\right. \\
& \left.+\frac{t}{3} \int_{\Omega} R-\frac{t}{6} \int_{\partial \Omega} J+O\left(t^{3 / 2}\right)\right) \text { as } t \rightarrow 0^{+},
\end{aligned}
$$

where $\beta_{k}^{-}$(respectively, $\beta_{k}^{+}$) is the $k$-th Dirichlet-Laplacian (respectively, Neumann-Laplacian) eigenvalue on $\Omega ; R$ and $J$ are the scalar curvature and the mean curvature of $\Omega$ and $\partial \Omega$, respectively; $O\left(t^{3 / 2}\right)$ cannot be improved. 
The symbolic approach by Seeley [68, 69] and Greiner [30] is a very powerful general analytical procedure for analyzing the structure of the asymptotic expansion based on the theory of pseudodifferential operators and the calculus of symbols of operators. This approach may be considered for calculation of the heat invariants explicitly in terms of the jets of the symbol of the operator; it provides an iterative procedure for such a calculation. However, as far as we know, because of the technical complexity and, most importantly, lack of the manifest covariance, such analytical tools have never been used for the actual calculation of the explicit form of the heat invariants in an invariant geometric form (see [10]). The systematic explicit calculation of heat kernel coefficients for Laplace type operators is now well understood due to the work of Gilkey [27] and many others (see [28, 26, 32, 33, 76, 42, 13, 5, 51, 4] and references therein) because the Riemannian structure on a manifold is determined by a Laplace type operator. For the classical boundary conditions, like Dirichlet, Neumann, Robin, and mixed combination thereof on vector bundles, the coefficients of the trace of heat kernel have been explicitly computed up to the first five terms (see, for example, [43, 14, 15, 6]). For other type operators (which originated from physics problems), the corresponding explicit form of the heat invariants have also been discussed. Liu in [52 explicitly calculated the first two coefficients of asymptotic expansion of the heat trace for the Stokes operator (i.e., incompressible slow flow operator), and in [53, 54] gave the first four coefficients of asymptotic expansion of the heat trace for the Dirichlet-to-Neumann map (We also refer the reader to [67] for the asymptotic expansion of the first three coefficients) as well as polyharmonic Steklov operator.

Contrary to the Laplace type operators, there are no systematic effective methods for an explicit calculation of the spectral invariants for second-order operators which are not of Laplace type. Such operators appear in so-called matrix geometry [7, 8, 9, when instead of a single Riemannian metric there is a matrix-valued symmetric 2-tensor. Let us point out that the Navier-Lamé operator is just a non-Laplace type operator. Five decades ago Greiner [30], p. 164, indicated that "the problem of interpreting these coefficients geometrically remains open". There has not been much progress in this direction. Thus, the geometric aspect of the spectral asymptotics of the Navier-Lamé operator remains an open problem (see [10]). In the celebrated paper [10], Avramidi consider general non-Laplace type operators on manifolds with boundary by introducing a "noncommutative" Dirac operator as a first-order elliptic partial differential operator such that its square is a second-order self-adjoint elliptic operator with positive definite leading symbol (not necessarily of Laplace type) and study the spectral asymptotics of these operators with Dirichlet boundary conditions. However, Avramidi's method is too complicated to be applied to the Navier-Lamé operator because of extremely technical difficulty to calculate the noncommutative Dirac operator and required symbol integral for such an operator.

In this paper, by combining the technique of calculus of symbols for the integral kernel and "method of images", we obtain the following result:

Theorem 1.1. Let $(\Omega, g)$ be a compact Riemannian manifold of dimension $n$ with smooth boundary $\partial \Omega$, and let $0<\tau_{1}^{-}<\tau_{2}^{-} \leq \cdots \leq \tau_{k}^{-} \leq \cdots$ (respectively, $0 \leq \tau_{1}^{+}<\tau_{2}^{+} \leq \tau_{3}^{+} \leq \cdots \leq \tau_{k}^{+} \leq \cdots$ ) be the eigenvalues of the Navier-Lamé operator $P_{g}^{-}$(respectively, $P_{g}^{+}$) with respect to the Dirichlet (respectively, Neumann) boundary condition. Then

$$
\begin{aligned}
& \sum_{k=1}^{\infty} e^{-\tau_{k}^{\mp} t}=\operatorname{Tr}\left(e^{-t P_{g}^{\mp}}\right)=\left[\frac{(n-1)}{(4 \pi \mu t)^{n / 2}}+\frac{1}{(4 \pi(2 \mu+\lambda) t)^{n / 2}}\right] \operatorname{Vol}(\Omega) \\
& \mp \frac{1}{4}\left[\frac{(n-1)}{(4 \pi \mu t)^{(n-1) / 2}}+\frac{1}{(4 \pi(2 \mu+\lambda) t)^{(n-1) / 2}}\right] \operatorname{Vol}(\partial \Omega)+O\left(t^{1-n / 2}\right) \quad \text { as } t \rightarrow 0^{+} .
\end{aligned}
$$

Here $\operatorname{Vol}(\Omega)$ denotes the $n$-dimensional volume of $\Omega, \operatorname{Vol}(\partial \Omega)$ denotes the $(n-1)$-dimensional volume of $\partial \Omega$.

Our result shows that not only the volume $\operatorname{Vol}(\Omega)$ but also the surface area $\operatorname{Vol}(\partial \Omega)$ can be obtained if we know all the Navier-Lamé eigenvalues with respect to the Dirichlet (respectively, Neumann) boundary condition. This gives an answer to an interesting and open problem mentioned by Avramidi in [10]. Roughly speaking, one can "hear" the volume of the domain and the surface area of its boundary by "hearing" all the pitches of the vibration of an elastic body. 
The key ideas of this paper are as follows. We denote by $\left(e^{-t P_{g}^{\mp}}\right)_{t \geq 0}$ the parabolic semigroups generated by $-P_{g}^{\mp}$. More precisely, $\mathbf{w}^{\mp}(t, x)=e^{-t P_{g}^{\mp}} \mathbf{w}_{0}(x)$ solve the following initial-boundary problems:

$$
\begin{cases}\frac{\partial \mathbf{w}^{-}}{\partial t}+\mu \nabla^{*} \nabla \mathbf{w}^{-}-(\mu+\lambda) \operatorname{grad} \operatorname{div} \mathbf{w}^{-}-\mu \operatorname{Ric}\left(\mathbf{w}^{-}\right)=0 & \text { in }(0,+\infty) \times \Omega \\ \mathbf{w}^{-}=0 & \text { on }(0,+\infty) \times \partial \Omega \\ \mathbf{w}^{-}(0, x)=\mathbf{w}_{0} & \text { on }\{0\} \times \Omega\end{cases}
$$

and

$$
\begin{cases}\frac{\partial \mathbf{w}^{+}}{\partial t}+\mu \nabla^{*} \nabla \mathbf{w}^{+}-(\mu+\lambda) \operatorname{grad} \operatorname{div} \mathbf{w}^{+}-\mu \operatorname{Ric}\left(\mathbf{w}^{+}\right)=0 & \text { in }(0,+\infty) \times \Omega \\ \frac{\partial \mathbf{w}^{+}}{\partial \nu}=0 & \text { on }(0,+\infty) \times \partial \Omega \\ \mathbf{w}^{+}(0, x)=\mathbf{w}_{0} & \text { on }\{0\} \times \Omega\end{cases}
$$

If $\left\{\mathbf{u}_{k}^{\mp}\right\}_{k=1}^{\infty}$ are orthonormal eigenvectors of the Navier-Lamé problem corresponding to eigenvalues $\left\{\tau_{k}^{\mp}\right\}_{k=1}^{\infty}$, then the integral kernels $\mathbf{K}^{\mp}(t, x, y)=e^{-t P_{g}^{\mp}} \delta(x-y)$ of the semigroups are given by

$$
\mathbf{K}^{\mp}(t, x, y)=\sum_{k=1}^{\infty} e^{-t \tau_{k}^{\mp}} \mathbf{u}_{k}^{\mp}(x) \otimes \mathbf{u}_{k}^{\mp}(y) .
$$

Thus the integrals of the traces of $\mathbf{K}^{\mp}(t, x, y)$ are actually spectral invariants:

$$
\int_{\Omega}\left(\operatorname{Tr}\left(\mathbf{K}^{\mp}(t, x, x)\right)\right) d V=\sum_{k=1}^{\infty} e^{-t \tau_{k}^{\mp}}
$$

To further analyze the geometric content of the spectrum, we calculate the same integral of the trace by another approach: let $\mathcal{M}=\Omega \cup(\partial \Omega) \cup \Omega^{*}$ be the (closed) double of $\Omega$, and $\mathcal{P}$ the double to $\mathcal{M}$ of the operator $P_{g}$ on $\Omega$ (see section 4 ). Then $-\mathcal{P}$ generates a strongly continuous semigroup $\left(e^{-t \mathcal{P}}\right)_{t \geq 0}$ on $L^{2}(\mathcal{M})$ with integral kernel $\mathbf{K}(t, x, y)$. Clearly, $\mathbf{K}^{\mp}(t, x, y)=\mathbf{K}(t, x, y) \mp \mathbf{K}(t, x, \stackrel{*}{y})$ for $x, y \in \bar{\Omega}$, where $\stackrel{*}{y}$ is the double of $y \in \Omega$. This technique stems from McKean and Singer (see [60]), and is called "method of images". Since $e^{-t \mathcal{P}} f(x)=\frac{1}{2 \pi i} \int_{\mathcal{C}} e^{-t \tau}(\tau I-\mathcal{P})^{-1} f(x) d \tau$, we have

$$
e^{-t \mathcal{P}} f(x)=\frac{1}{(2 \pi)^{n}} \int_{\mathbb{R}^{n}} e^{i x \cdot \xi}\left(\frac{1}{2 \pi i} \int_{\mathcal{C}} e^{-t \tau} \iota\left((\tau I-\mathcal{P})^{-1}\right) \hat{f}(\xi) d \tau\right) d \xi
$$

so that

$$
\begin{aligned}
\mathbf{K}(t, x, y) & =e^{-t \mathcal{P}} \delta(x-y)=\frac{1}{(2 \pi)^{n}} \int_{\mathbb{R}^{n}} e^{i(x-y) \cdot \xi}\left(\frac{1}{2 \pi i} \int_{\mathcal{C}} e^{-t \tau} \iota\left((\tau I-\mathcal{P})^{-1}\right) d \tau\right) d \xi \\
& =\frac{1}{(2 \pi)^{n}} \int_{\mathbb{R}^{n}} e^{i(x-y) \cdot \xi}\left(\frac{1}{2 \pi i} \int_{\mathcal{C}} e^{-t \tau}\left(\sum_{l \geq 0} \mathbf{q}_{-2-l}(x, \xi, \tau)\right) d \tau\right) d \xi
\end{aligned}
$$

where $\mathcal{C}$ is a suitable curve in the complex plane in the positive direction around the spectrum of $\mathcal{P}$, and $\iota\left((\tau I-\mathcal{P})^{-1}\right):=\sum_{l \geq 0} \mathbf{q}_{-2-l}(x, \xi, \tau)$ is the full symbol of resolvent operator $(\tau I-\mathcal{P})^{-1}$. This implies that for any $x \in \bar{\Omega}$,

$$
\begin{aligned}
& \operatorname{Tr}(\mathbf{K}(t, x, x))=\frac{1}{(2 \pi)^{n}} \int_{\mathbb{R}^{n}}\left(\frac{1}{2 \pi i} \int_{\mathcal{C}} e^{-t \tau} \sum_{l \geq 0} \operatorname{Tr}\left(\mathbf{q}_{-2-l}(x, \xi, \tau)\right) d \tau\right) d \xi \\
& \operatorname{Tr}(\mathbf{K}(t, x, \stackrel{*}{x}))=\frac{1}{(2 \pi)^{n}} \int_{\mathbb{R}^{n}} e^{i(x-*) \cdot \xi}\left(\frac{1}{2 \pi i} \int_{\mathcal{C}} e^{-t \tau} \sum_{l \geq 0} \operatorname{Tr}\left(\mathbf{q}_{-2-l}(x, \xi, \tau)\right) d \tau\right) d \xi .
\end{aligned}
$$

It is easy to show that for any $x \in \Omega$,

$$
\begin{aligned}
& \frac{1}{(2 \pi)^{n}} \int_{\mathbb{R}^{n}}\left(\frac{1}{2 \pi i} \int_{\mathcal{C}} e^{-t \tau} \sum_{l \geq 1} \operatorname{Tr}\left(\mathbf{q}_{-2-l}(x, \xi, \tau)\right) d \tau\right) d \xi=O\left(t^{1-\frac{n}{2}}\right) \quad \text { as } t \rightarrow 0^{+}, \\
& \frac{1}{(2 \pi)^{n}} \int_{\mathbb{R}^{n}} e^{i(x-x) \cdot \xi}\left(\frac{1}{2 \pi i} \int_{\mathcal{C}} e^{-t \tau} \sum_{l \geq 1} \operatorname{Tr}\left(\mathbf{q}_{-2-l}(x, \xi, \tau)\right) d \tau\right) d \xi=O\left(t^{1-\frac{n}{2}}\right) \quad \text { as } t \rightarrow 0^{+} .
\end{aligned}
$$


In order to finally establish asymptotic estimate, we denote by $U_{\epsilon}(\partial \Omega)$ the $\epsilon$-neighborhood of $\partial \Omega$ in $\mathcal{M}$. We can also show that

$$
\begin{aligned}
& \frac{1}{(2 \pi)^{n}} \int_{\mathbb{R}^{n}}\left(\frac{1}{2 \pi i} \int_{\mathcal{C}} \mathbf{q}_{-2}(x, \xi, \tau) d \tau\right) d \xi=\left(\frac{n-1}{(4 \pi \mu t)^{n / 2}}+\frac{1}{(4 \pi(2 \mu+\lambda) t)^{n / 2}}\right) \quad \text { for } x \in \Omega, \\
& \frac{1}{(2 \pi)^{n}} \int_{\mathbb{R}^{n}} e^{i(x-*) \cdot \xi}\left(\frac{1}{2 \pi i} \int_{\mathcal{C}} \mathbf{q}_{-2}(x, \xi, \tau) d \tau\right) d \xi=O\left(t^{1-\frac{n}{2}}\right) \quad \text { as } t \rightarrow 0^{+} \text {for } x \in \Omega \backslash U_{\epsilon}(\partial \Omega)
\end{aligned}
$$

and

$$
\begin{aligned}
\int_{\Omega \cap U_{\epsilon}(\partial \Omega)} & \left\{\frac{1}{(2 \pi)^{n}} \int_{\mathbb{R}^{n}} e^{i(x-x) \cdot \xi}\left(\frac{1}{2 \pi i} \int_{\mathcal{C}} \mathbf{q}_{-2}(x, \xi, \tau) d \tau\right) d \xi\right\} d V \\
= & \frac{1}{4}\left(\frac{n-1}{(4 \pi \mu t)^{(n-1) / 2}}+\frac{1}{(4 \pi(2 \mu+\lambda) t)^{(n-1) / 2}}\right) \operatorname{Vol}(\partial \Omega)+O\left(t^{1-\frac{n}{2}}\right) \quad \text { as } t \rightarrow 0^{+} .
\end{aligned}
$$

Hence

$$
\int_{\Omega} \operatorname{Tr}\left(\mathbf{K}^{\mp}(t, x, x)\right) d V=a_{0} t^{-n / 2} \mp a_{1} t^{-(n-1) / 2}+O\left(t^{1-n / 2}\right) \quad \text { as } t \rightarrow 0^{+},
$$

where $a_{0}=\left(\frac{n-1}{(4 \pi \mu)^{n / 2}}+\frac{1}{(4 \pi(2 \mu+\lambda))^{n / 2}}\right) \operatorname{Vol}(\Omega)$ and $a_{1}=\frac{1}{4}\left(\frac{(n-1)}{(4 \pi \mu)^{(n-1) / 2}}+\frac{1}{(4 \pi(2 \mu+\lambda))^{(n-1) / 2}}\right) \operatorname{Vol}(\partial \Omega)$.

As an application of theorem 1.1, we can prove the following spectral rigidity result:

Theorem 1.2. Let $\Omega \subset \mathbb{R}^{n}$ be a bounded domain with smooth boundary $\partial \Omega$. Suppose that the Navier-Lamé spectrum with respect to the Dirichlet (respectively, Neumann) boundary condition, is equal to that of $B_{r}, a$ ball of radius $r$. Then $\Omega=B_{r}$.

Theorem 1.2 also shows that a ball is uniquely determined by its Navier-Lamé spectrum among all Euclidean bounded domains (elastic bodies) with smooth boundary.

\section{Some nOtATiOnS AND LEMmas}

Let $\Omega$ be an $n$-dimensional Riemannian manifold (possibly with boundary), and let $\Omega$ be equipped with a smooth metric tensor $g=\sum_{j, k=1}^{n} g_{j k} d x_{j} \otimes d x_{k}$. Denote by $\left[g^{j k}\right]_{n \times n}$ the inverse of the matrix $\left[g_{j k}\right]_{n \times n}$ and set $|g|:=\operatorname{det}\left[g_{j k}\right]_{n \times n}$. In particular, $d \mathrm{~V}$, the volume element in $\Omega$ is locally given by $d \mathrm{~V}=\sqrt{|g|} d x_{1} \cdots d x_{n}$. By $T \Omega$ and $T^{*} \Omega$ we denote, respectively, the tangent and cotangent bundle on $\Omega$. Throughout, we shall also denote by $T \Omega$ global $\left(C^{\infty}\right)$ sections in $T \Omega$ (i.e., $T \Omega \equiv C^{\infty}(\Omega, T \Omega)$ ); similarly, $T^{*} \Omega \equiv C^{\infty}\left(\Omega, T^{*} \Omega\right)$. A vector field on $\Omega$ is a section of the map $\pi: T \Omega \rightarrow \Omega$. More concretely, a vector field is a smooth map $X: \Omega \rightarrow T \Omega$, usually written $p \mapsto X_{p}$, with the property that

$$
\pi \circ X=\mathrm{Id}_{\Omega},
$$

or equivalently, $X_{p} \in T_{p} \Omega$ for each $p \in \Omega$. If $\left(U ; x_{1}, \cdots, x_{n}\right)$ is any smooth coordinate chart for $\Omega$, we can write the value of $X$ at any point $p \in U$ in terms of the coordinate basis vectors $\left\{\left.\frac{\partial}{\partial x_{j}}\right|_{p}\right\}$ of $T_{p} \Omega$ :

$$
X_{p}=\left.\sum_{j=1}^{n} X^{j}(p) \frac{\partial}{\partial x_{j}}\right|_{p}
$$

This defines $n$ functions $X^{j}: U \rightarrow \mathbb{R}$, called the component functions of $X$ in the given chart. Recall first that

$$
\operatorname{div} X:=\sum_{j=1}^{n} \frac{1}{\sqrt{|g|}} \frac{\partial\left(\sqrt{|g|} X^{j}\right)}{\partial x_{j}} \quad \text { if } X=\sum_{j=1}^{n} X^{j} \frac{\partial}{\partial x_{j}} \in T \Omega
$$


and

$$
\operatorname{grad} v=\sum_{j, k=1}^{n}\left(g^{j k} \frac{\partial v}{\partial x_{k}}\right) \frac{\partial}{\partial x_{j}} \quad \text { if } v \in C^{\infty}(\Omega),
$$

are, respectively, the usual divergence and gradient operators. Accordingly, the Laplace-Beltrami operator $\Delta_{g}$ is just given by

$$
\Delta_{g}:=\operatorname{div} \operatorname{grad}=\frac{1}{\sqrt{|g|}} \sum_{j, k=1}^{n} \frac{\partial}{\partial x_{j}}\left(\sqrt{|g|} g^{j k} \frac{\partial}{\partial x_{k}}\right) .
$$

Next, let $\nabla$ be the associated Levi-Civita connection. For each $X \in T \Omega, \nabla X$ is the tensor of type $(0,2)$ defined by

$$
(\nabla X)(Y, Z):=\left\langle\nabla_{Z} X, Y\right\rangle, \quad \forall Y, Z \in T \Omega .
$$

It is well-known that in a local coordinate system with the naturally associated frame field on the tangent bundle,

$$
\nabla_{\frac{\partial}{\partial x_{k}}} X=\sum_{j=1}^{n}\left(\frac{\partial X^{j}}{\partial x_{k}}+\sum_{l=1}^{n} \Gamma_{l k}^{j} X^{l}\right) \frac{\partial}{\partial x_{j}} \quad \text { for } X=\sum_{j=1}^{n} X^{j} \frac{\partial}{\partial x_{j}},
$$

where $\Gamma_{l k}^{j}=\frac{1}{2} \sum_{m=1}^{n} g^{j m}\left(\frac{\partial g_{k m}}{\partial x_{l}}+\frac{\partial g_{l m}}{\partial x_{k}}-\frac{\partial g_{l k}}{\partial x_{m}}\right)$ are the Christoffel symbols associated with the metric $g$ (see, for example, p. 549 of [75]). If we denote

$$
X_{; k}^{j}=\frac{\partial X^{j}}{\partial x_{k}}+\sum_{l=1}^{n} \Gamma_{l k}^{j} X^{l}
$$

then

$$
\nabla_{Y} X=\sum_{j, k=1}^{n} Y^{k} X_{; k}^{j} \frac{\partial}{\partial x_{j}} \text { for } Y=\sum_{k=1}^{n} Y^{k} \frac{\partial}{\partial x_{k}} .
$$

The symmetric part of $\nabla X$ is $\operatorname{Def} X$, the deformation of $X$, i.e.,

$$
(\operatorname{Def} X)(Y, Z)=\frac{1}{2}\left\{\left\langle\nabla_{Y} X, Z\right\rangle+\left\langle\nabla_{Z} X, Y\right\rangle\right\}, \quad \forall Y, Z \in T \Omega
$$

(whereas the antisymmetric part of $\nabla X$ is simply $d X$, i.e.,

$$
\left.d X(Y, Z)=\frac{1}{2}\left\{\left\langle\nabla_{Y} X, Z\right\rangle-\left\langle\nabla_{Z} X, Y\right\rangle\right\}, \quad \forall Y, Z \in T \Omega .\right)
$$

Thus, Def $X$ is a symmetric tensor field of type $(0,2)$. In coordinate notation,

$$
(\operatorname{Def} X)_{j k}=\frac{1}{2}\left(X_{j ; k}+X_{k ; j}\right), \quad \forall j, k,
$$

where, $X_{k ; j}:=\frac{\partial X_{k}}{\partial \mathbf{x}_{j}}-\sum_{l=1}^{n} \Gamma_{k j}^{l} X_{l}$ for a vector field $X=\sum_{j=1}^{n} X^{j} \frac{\partial}{\partial x_{j}}$, and $X_{k}=\sum_{l=1}^{n} g_{k l} X^{l}$. The adjoint Def* of Def is defined in local coordinates by $\left(\operatorname{Def}^{*} w\right)^{j}=-\sum_{k=1}^{n} w_{; k}^{j k}$ for each symmetric tensor field $w:=w_{j k}$ of type $(0,2)$. The Riemann curvature tensor $\mathcal{R}$ of $\Omega$ is given by

$$
\mathcal{R}(X, Y) Z=\left[\nabla_{X}, \nabla_{Y}\right] Z-\nabla_{[X, Y]} Z, \quad \forall X, Y, Z \in T \Omega,
$$

where $[X, Y]:=X Y-Y X$ is the usual commutator bracket. It is convenient to change this into a $(0,4)$-tensor by setting

$$
\mathcal{R}(X, Y, Z, W):=\langle\mathcal{R}(X, Y) Z, W\rangle, \quad \forall X, Y, Z, W \in T \Omega .
$$

In other words, in a local coordinate system such as that discussed above,

$$
R_{j k l m}=\left\langle\mathcal{R}\left(\frac{\partial}{\partial x_{l}}, \frac{\partial}{\partial x_{m}}\right) \frac{\partial}{\partial x_{k}}, \frac{\partial}{\partial x_{j}}\right\rangle .
$$


The Ricci curvature Ric on $\Omega$ is a $(0,2)$-tensor defined as a contraction of $\mathcal{R}$ :

$$
\operatorname{Ric}(X, Y):=\sum_{j=1}^{n}\left\langle\mathcal{R}\left(\frac{\partial}{\partial x_{j}}, Y\right) X, \frac{\partial}{\partial x_{j}}\right\rangle=\sum_{j=1}^{n}\left\langle\mathcal{R}\left(Y, \frac{\partial}{\partial x_{j}}\right) \frac{\partial}{\partial x_{j}}, X\right\rangle, \quad \forall X, Y \in T \Omega .
$$

That is,

$$
R_{j k}=\sum_{l=1}^{n} R_{j l k}^{l}=\sum_{l, m=1}^{n} g^{l m} R_{l j m k}
$$

Note that

$$
R_{j l k}^{l}=\frac{\partial \Gamma_{j k}^{l}}{\partial x_{l}}-\frac{\partial \Gamma_{j l}^{l}}{\partial x_{k}}+\sum_{s=1}^{n}\left(\Gamma_{s l}^{l} \Gamma_{j k}^{s}-\Gamma_{s k}^{l} \Gamma_{j l}^{s}\right)
$$

In [56], by considering the equilibria states of elastic energy functional $\mathcal{E}(\mathbf{u})=-\frac{1}{2} \int_{\Omega}\left(\lambda(\operatorname{div} \mathbf{u})^{2}+2 \mu\langle\operatorname{Def} \mathbf{u}\right.$, Def $\left.\mathbf{u}\rangle\right) d V$, the author of this paper proved the following result, which generalizes the classical Navier-Lamé operator from the Euclidean space to a Riemannian manifold:

Lemma 2.1. On a Riemannian manifold $(\Omega, g)$, modeling a homogeneous, linear, isotropic, elastic medium, the Navier-Lamé operator $\mathcal{L}_{g}$ is given by

$$
P_{g} \mathbf{u}=\mu \nabla^{*} \nabla \mathbf{u}-(\mu+\lambda) \operatorname{grad} \operatorname{div} \mathbf{u}-\mu \operatorname{Ric}(\mathbf{u}) \quad \text { for } \mathbf{u}=\sum_{k=1}^{n} u^{k} \frac{\partial}{\partial x_{k}} \in T \Omega,
$$

where $\nabla^{*} \nabla \mathbf{u}$ is the Bochner Laplacian of $\mathbf{u}$ defined by

$$
\nabla^{*} \nabla \mathbf{u}=-\sum_{j=1}^{n}\left\{\Delta_{g} u^{j}+2 \sum_{m, k, l=1}^{n} g^{m l} \Gamma_{k m}^{j} \frac{\partial u^{k}}{\partial x_{l}}+\sum_{m, k, l=1}^{n}\left(g^{m l} \frac{\partial \Gamma_{k l}^{j}}{\partial x_{m}}+\sum_{h=1}^{n} g^{m l} \Gamma_{h l}^{j} \Gamma_{k m}^{h}-\sum_{h=1}^{n} g^{m l} \Gamma_{k h}^{j} \Gamma_{m l}^{h}\right) u^{k}\right\} \frac{\partial}{\partial x_{j}},
$$

and

$$
\operatorname{Ric}(\mathbf{u})=\sum_{j=1}^{n}\left(\sum_{k=1}^{n} R_{k}^{j} u^{k}\right) \frac{\partial}{\partial x_{j}}
$$

In particular, $P_{g}$ is strongly elliptic, formally self-adjoint.

We need the method of pseudodifferential operators. If $W$ is an open subset of $\mathbb{R}^{n}$, we denote by $S_{1,0}^{m}=$ $S_{1,0}^{m}\left(W, \mathbb{R}^{n}\right)$ the set of all $p \in C^{\infty}\left(W, \mathbb{R}^{n}\right)$ such that for every compact set $O \subset W$ we have

$$
\left|D_{x}^{\beta} D_{\xi}^{\alpha} p(x, \xi)\right| \leq C_{O, \alpha, \beta}(1+|\xi|)^{m-|\alpha|}, \quad x \in O, \xi \in \mathbb{R}^{n}
$$

for all $\alpha, \beta \in \mathbb{N}^{n}$, where $|\xi|=\left(\sum_{j=1}^{n} \xi_{j}^{2}\right)^{1 / 2}, D^{\alpha}=D_{1}^{\alpha_{1}} \cdots D_{n}^{\alpha_{n}}, D_{j}=\frac{1}{i} \frac{\partial}{\partial x_{j}}, \mathbb{N}^{n}$ is the set of $\gamma=\left(\gamma_{1}, \cdots, \gamma_{n}\right)$ with $\gamma_{j}=$ integer $\geq 0$, and $|\gamma|=\gamma_{1}+\cdots+\gamma_{n}$. The elements of $S_{1,0}^{m}$ are called symbols (or full symbols) of order $m$. It is clear that $S_{1,0}^{m}$ is a Fréchet space with semi-norms given by the smallest constants which can be used in (2.13) (i.e.,

$$
\left.\|p\|_{O, \alpha, \beta}=\sup _{x \in O}\left|\left(D_{x}^{\beta} D_{\xi}^{\alpha} p(x, \xi)\right)(1+|\xi|)^{|\alpha|-m}\right|\right) .
$$

Let $p(x, \xi) \in S_{1,0}^{m}$. A pseudodifferential operator in an open set $W \subset \mathbb{R}^{n}$ is essentially defined by a Fourier integral operator (cf. [44, [37, 75], 32]):

$$
p(x, D) u(x)=\frac{1}{(2 \pi)^{n}} \int_{\mathbb{R}^{n}} e^{i x \cdot \xi} p(x, \xi) \hat{u}(\xi) d \xi
$$

and denoted by $O P S^{m}$. Here $u \in C_{0}^{\infty}(W)$ and $\hat{u}(\xi)=\int_{\mathbb{R}^{n}} e^{-i y \cdot \xi} u(y) d y$ is the Fourier transform of $u$. If there are smooth $p_{m-j}(x, \xi)$, homogeneous in $\xi$ of degree $m-j$ for $|\xi| \geq 1$, that is, $p_{m-j}(x, r \xi)=r^{m-j} p_{m-j}(x, \xi)$ for $r,|\xi| \geq 1$, and if

$$
p(x, \xi) \sim \sum_{j \geq 0} p_{m-j}(x, \xi)
$$


in the sense that

$$
p(x, \xi)-\sum_{j=0}^{k} p_{m-j}(x, \xi) \in S_{1,0}^{m-k-1}
$$

for all $k$, then we say $p(x, \xi) \in S_{c l}^{m}$, or just $p(x, \xi) \in S^{m}$. We call $p_{m}(x, \xi)$ the principal symbol of $p(x, D)$. Sometimes we denote by $\iota(p(x, D))$ the (full) symbol of $p(x, D)$.

Let $\Omega$ be a smooth $n$-dimensional Riemannian manifold (of class $C^{\infty}$ ). We denote by $C^{\infty}(\Omega)$ and $C_{0}^{\infty}(\Omega)$ the space of all smooth complex-valued functions on $\Omega$ and the subspace of all functions with compact support, respectively. Assume that we are given a linear operator

$$
P: C_{0}^{\infty}(\Omega) \rightarrow C^{\infty}(\Omega) .
$$

If $G$ is some chart in $\Omega$ (not necessarily connected) and $\kappa: G \rightarrow U$ its diffeomorphism onto an open set $U \subset \mathbb{R}^{n}$, then let $\tilde{P}$ be defined by the diagram

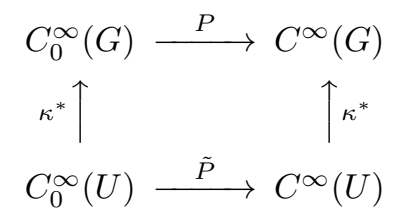

where $\kappa^{*}$ is the induced transformation from $C^{\infty}(U)$ into $C^{\infty}(G)$, taking a function $u$ to the function $u \circ \kappa$. (note, in the upper row is the operator $r_{G} \circ P \circ i_{G}$, where $i_{G}$ is the natural embedding $i_{G}: C_{0}^{\infty}(G) \rightarrow C_{0}^{\infty}(\Omega)$ and $r_{G}$ is the natural restriction $r_{G}: C^{\infty}(\Omega) \rightarrow C^{\infty}(G)$; for brevity we denote this operator by the same letter $P$ as the original operator). An operator $P: C_{0}^{\infty}(\Omega) \rightarrow C^{\infty}(\Omega)$ is called a pseudodifferential operator on $\Omega$ if for any chart diffeomorphism $\kappa: G \rightarrow U$, the operator $\tilde{P}$ defined above is a pseudodifferential operator on $U$. We denote by $O P S^{m}$ the pseudodifferential operator $P$ of order $m$. We also write $O P S^{-\infty}=\bigcap_{m} O P S^{m}$.

It is well known (see [36, 37] or p. 13 of [75]) that if $p_{j}(x, D) \in O P S^{m_{j}}, j=1,2$, then

$$
p_{1}(x, D) p_{2}(x, D)=q(x, D) \in O P S^{m_{1}+m_{2}},
$$

and

$$
q(x, \xi)=\sum_{\alpha \geq 0} \frac{i^{|\alpha|}}{\alpha !} D_{\xi}^{\alpha} p_{1}(x, \xi) D_{x}^{\alpha} p_{2}(x, \xi) .
$$

An operator $p(x, D)$ is said to be an elliptic pseudodifferential operator of order $m$ if for every compact $O \subset \Omega$ there exists a positive constant $c=c(O)$ such that

$$
|p(x, \xi)| \geq c|\xi|^{m}, x \in O,|\xi| \geq 1 .
$$

If $q(x, D) \in O P S^{-m}$ is a pseudodifferential operator of order $-m$ such that

$$
\begin{aligned}
& q(x, D) p(x, D)=I \bmod O P S^{-\infty}, \\
& p(x, D) q(x, D)=I \bmod O P S^{-\infty},
\end{aligned}
$$

then we say that $q(x, D)$ is a (two-sided) parametrix for $p(x, D)$. Furthermore, if $P$ is a non-negative elliptic pseudodifferential operator of order $m$, then the spectrum of $P$ lies in a right half-plane and has a finite lower bound $\rho(P)=\inf \{\operatorname{Re} \tau \mid \tau \in \sigma(P)\}$, where $\sigma(P)$ denotes the spectrum of $P$. We can modify the principal symbol $h_{m}(x, \xi)$ for small $\xi$ such that $h_{m}(x, \xi)$ has a positive lower bound throughout and lies in $\left\{\tau=r e^{i \theta}|r>0,| \theta \mid \leq \theta_{0}\right\}$, where $\theta_{0} \in\left(0, \frac{\pi}{2}\right)$. According to [32], the resolvent $(\tau-P)^{-1}$ exists and is holomorphic in $\tau$ on a neighborhood of a set

$$
W_{r_{0}, \epsilon}=\left\{\tau \in \mathbb{C}|| \tau \mid \geq r_{0}, \arg \tau \in\left[\theta_{0}+\epsilon, 2 \pi-\theta_{0}-\epsilon\right], \operatorname{Re} \tau \leq \rho(P)-\epsilon\right\}
$$

(with $\epsilon>0$ ). There exists a parametrix $Q_{\tau}^{\prime}$ on a neighborhood of a possibly larger set (with $\delta>0, \epsilon>0$ )

$$
V_{\delta, \epsilon}=\left\{\tau \in \mathbb{C}|| \tau \mid \geq \delta \text { or } \arg \tau \in\left[\theta_{0}+\epsilon, 2 \pi-\theta_{0}-\epsilon\right]\right\}
$$

such that this parametrix coincides with $(\tau-P)^{-1}$ on the intersection. Its symbol $q(x, \xi, \tau)$ in local coordinates is holomorphic in $\tau$ there and has the form (cf. Section 3.3 of [32])

$$
q(x, \xi, \tau) \sim \sum_{l \geq 0} q_{-m-l}(x, \xi, \tau),
$$


where

$$
\begin{gathered}
q_{-m}=\left(\tau-p_{m}(x, \xi)\right)^{-1}, \quad q_{-m-1}=b_{1,1}(x, \xi) q_{-m}^{2} \\
\cdots, q_{-m-l}=\sum_{k=1}^{2 l} b_{l, k}(x, \xi) q_{-m}^{k+1}, \cdots, l \geq 2
\end{gathered}
$$

with symbols $b_{l, k}$ independent of $\tau$ and homogeneous of degree $m k-l$ in $\xi$ for $|\xi| \geq 1$. The semigroup $\left(e^{-t P}\right)_{t \geq 0}$ can be defined from $P$ by the Cauchy integral formula (see p. 4 of [29]):

$$
e^{-t P}=\frac{1}{2 \pi i} \int_{\mathcal{C}} e^{-t \tau}(\tau-P)^{-1} d \tau, \quad t \geq 0
$$

where $\mathcal{C}$ is a suitable curve in the complex plane in the positive direction around the spectrum of $P$. Inserting (2.18) - 2.19) into above formula, we get the symbol $\frac{1}{2 \pi i} \int_{\mathcal{C}} e^{-t \tau}\left[\sum_{l \geq 0} q_{-m-l}(x, \xi, \tau)\right] d \tau$ of the semigroup $\left(e^{-t P}\right)_{t \geq 0}$, and furthermore we can obtain the semigroup $\left(e^{-t P}\right)_{t \geq 0}$ and its trace for any fixed $t \geq 0$.

\section{Full symbol of Resolvent operator $\left(\tau I-P_{g}\right)^{-1}$}

Let $(\Omega, g)$ be an $n$-dimensional Riemannian manifold with metric $g=\left(g_{i j}\right)$. Note that for $\mathbf{u}=\sum_{j=1}^{n} u^{j} \frac{\partial}{\partial x_{j}} \in$ $T \Omega$,

$$
\Delta_{g} u^{j}=\sum_{m, l=1}^{n}\left(g^{m l} \frac{\partial^{2} u^{j}}{\partial x_{m} \partial x_{l}}-\sum_{s=1}^{n} g^{m l} \Gamma_{m l}^{s} \frac{\partial u^{j}}{\partial x_{s}}\right)
$$

and

$$
\operatorname{grad} \operatorname{div} \mathbf{u}=\sum_{j, k, m=1}^{n}\left(g^{j m}\left(\frac{\partial^{2} u^{k}}{\partial x_{m} \partial x_{k}}+\sum_{l=1}^{n} \Gamma_{k l}^{l} \frac{\partial u^{k}}{\partial x_{m}}+\sum_{l=1}^{n} \frac{\partial \Gamma_{k l}^{l}}{\partial x_{m}} u^{k}\right)\right) \frac{\partial}{\partial x_{j}}
$$

By Lemma 2.1 we can write the Navier-Lamé operator $P_{g}$ in $\Omega$ as the form of components relative to coordinates:

$$
\begin{aligned}
& P_{g} \mathbf{u}=\left\{-\mu\left(\sum_{m, l=1}^{n} g^{m l} \frac{\partial^{2}}{\partial x_{m} \partial x_{l}}\right) \mathbf{I}_{n}-(\mu+\lambda)\left[\begin{array}{ccc}
\sum_{m=1}^{n} g^{1 m} \frac{\partial^{2}}{\partial x_{m} \partial x_{1}} & \cdots & \sum_{m=1}^{n} g^{1 m} \frac{\partial^{2}}{\partial x_{m} \partial x_{n}} \\
\vdots & \vdots \\
\sum_{m=1}^{n} g^{n m} \frac{\partial^{2}}{\partial x_{m} \partial x_{1}} & \cdots & \sum_{m=1}^{n} g^{n m} \frac{\partial^{2}}{\partial x_{m} \partial x_{n}}
\end{array}\right]\right. \\
& +\mu\left(\sum_{m, l, s=1}^{n} g^{m l} \Gamma_{m l}^{s} \frac{\partial}{\partial x_{s}}\right) \mathbf{I}_{n}-\mu\left[\begin{array}{ccc}
\sum_{m, l=1}^{n} 2 g^{m l} \Gamma_{1 m}^{1} \frac{\partial}{\partial x_{l}} & \cdots & \sum_{m, l=1}^{n} 2 g^{m l} \Gamma_{n m}^{1} \frac{\partial}{\partial x_{l}} \\
\vdots & \vdots \\
\sum_{m, l=1}^{n} 2 g^{m l} \Gamma_{1 m}^{n} \frac{\partial}{\partial x_{l}} & \cdots & \sum_{m, l=1}^{n} 2 g^{m l} \Gamma_{n m}^{n} \frac{\partial}{\partial x_{l}}
\end{array}\right] \\
& -(\mu+\lambda)\left[\begin{array}{ccc}
\sum_{m, l=1}^{n} g^{1 m} \Gamma_{1 l}^{l} \frac{\partial}{\partial x_{m}} & \cdots & \sum_{m, l=1}^{n} g^{1 m} \Gamma_{n l}^{l} \frac{\partial}{\partial x_{m}} \\
\vdots & & \vdots \\
\sum_{m, l=1}^{n} g^{n m} \Gamma_{1 l}^{l} \frac{\partial}{\partial x_{m}} & \cdots & \sum_{m, l=1}^{n} g^{n m} \Gamma_{n l}^{l} \frac{\partial}{\partial x_{m}}
\end{array}\right] \\
& -\mu\left[\begin{array}{ccc}
\sum_{l, m=1}^{n} g^{m l}\left(\frac{\partial \Gamma_{1 l}^{1}}{\partial x_{m}}+\Gamma_{h l}^{1} \Gamma_{1 m}^{h}-\Gamma_{1 h}^{1} \Gamma_{m l}^{h}\right) & \ldots & \sum_{l, m=1}^{n} g^{m l}\left(\frac{\partial \Gamma_{n l}^{1}}{\partial x_{m}}+\Gamma_{h l}^{1} \Gamma_{n m}^{h}-\Gamma_{n h}^{1} \Gamma_{m l}^{h}\right) \\
\vdots & \vdots \\
\sum_{l, m=1}^{n} g^{m l}\left(\frac{\partial \Gamma_{1 l}^{n}}{\partial x_{m}}+\Gamma_{h l}^{n} \Gamma_{1 m}^{h}-\Gamma_{1 h}^{n} \Gamma_{m l}^{h}\right) & \cdots & \sum_{l, m=1}^{n} g^{m l}\left(\frac{\partial \Gamma_{n l}^{n}}{\partial x_{m}}+\Gamma_{h l}^{n} \Gamma_{n m}^{h}-\Gamma_{n h}^{n} \Gamma_{m l}^{h}\right)
\end{array}\right]
\end{aligned}
$$




$$
\left.-(\mu+\lambda)\left[\begin{array}{ccc}
\sum_{l, m=1}^{n} g^{1 m} \frac{\partial \Gamma_{1 l}^{l}}{\partial x_{m}} & \cdots & \sum_{l, m=1}^{n} g^{1 m} \frac{\partial \Gamma_{n l}^{l}}{\partial x_{m}} \\
\vdots & & \vdots \\
\sum_{l, m=1}^{n} g^{n m} \frac{\partial \Gamma_{1 l}^{l}}{\partial x_{m}} & \cdots & \sum_{l, m=1}^{n} g^{n m} \frac{\partial \Gamma_{n l}^{l}}{\partial x_{m}}
\end{array}\right]-\mu\left[\begin{array}{ccc}
R_{1}^{1} & \cdots & R_{n}^{1} \\
\vdots & & \vdots \\
R_{1}^{n} & \cdots & R_{n}^{n}
\end{array}\right]\right\}\left[\begin{array}{c}
u^{1} \\
\vdots \\
u^{n}
\end{array}\right],
$$

where $\mathbf{I}_{n}$ is the $n \times n$ identity matrix. Furthermore, we have

$$
P_{g} \mathbf{u}(x)=\frac{1}{(2 \pi)^{n}} \int_{\mathbb{R}^{n}} e^{i x \cdot \xi}\left(\mathbf{A}_{g}(x, \xi)\right)\left(\begin{array}{c}
\hat{u}_{1}(\xi) \\
\vdots \\
\hat{u}_{n}(\xi)
\end{array}\right) d \xi
$$

where

$$
\begin{aligned}
& \mathbf{A}_{g}(x, \xi)=\mu\left(\sum_{m, l=1}^{n} g^{m l} \xi_{m} \xi_{l}\right) \mathbf{I}_{n}+(\mu+\lambda)\left[\begin{array}{ccc}
\sum_{m=1}^{n} g^{1 m} \xi_{m} \xi_{1} & \cdots & \sum_{m=1}^{n} g^{1 m} \xi_{m} \xi_{n} \\
\vdots & & \vdots \\
\sum_{m=1}^{n} g^{n m} \xi_{m} \xi_{1} & \cdots & \sum_{m=1}^{n} g^{n m} \xi_{m} \xi_{n}
\end{array}\right] \\
& +\mathrm{i} \mu\left(\sum_{m, l, s=1}^{n} g^{m l} \Gamma_{m l}^{s} \xi_{s}\right) \mathbf{I}_{n}-\mathrm{i} \mu\left[\begin{array}{ccc}
\sum_{m, l=1}^{n} 2 g^{m l} \Gamma_{1 m}^{1} \xi_{l} & \cdots & \sum_{m, l=1}^{n} 2 g^{m l} \Gamma_{n m}^{1} \xi_{l} \\
\vdots & & \vdots \\
\sum_{m, l=1}^{n} 2 g^{m l} \Gamma_{1 m}^{n} \xi_{l} & \cdots & \sum_{m, l=1}^{n} 2 g^{m l} \Gamma_{n m}^{n} \xi_{l}
\end{array}\right] \\
& -\mathrm{i}(\mu+\lambda)\left[\begin{array}{ccc}
\sum_{m, l=1}^{n} g^{1 m} \Gamma_{1 l}^{l} \xi_{m} & \cdots & \sum_{m, l=1}^{n} g^{1 m} \Gamma_{n l}^{l} \xi_{m} \\
\vdots & & \vdots \\
\sum_{m, l=1}^{n} g^{n m} \Gamma_{1 l}^{l} \xi_{m} & \cdots & \sum_{m, l=1}^{n} g^{n m} \Gamma_{n l}^{l} \xi_{m}
\end{array}\right] \\
& -\mu\left[\begin{array}{ccc}
\sum_{l, m=1}^{n} g^{m l}\left(\frac{\partial \Gamma_{1 l}^{1}}{\partial x_{m}}+\Gamma_{h l}^{1} \Gamma_{1 m}^{h}-\Gamma_{1 h}^{1} \Gamma_{m l}^{h}\right) & \ldots & \sum_{l, m=1}^{n} g^{m l}\left(\frac{\partial \Gamma_{n l}^{1}}{\partial x_{m}}+\Gamma_{h l}^{1} \Gamma_{n m}^{h}-\Gamma_{n h}^{1} \Gamma_{m l}^{h}\right) \\
\vdots & & \vdots \\
\sum_{l, m=1}^{n} g^{m l}\left(\frac{\partial \Gamma_{1 l}^{n}}{\partial x_{m}}+\Gamma_{h l}^{n} \Gamma_{1 m}^{h}-\Gamma_{1 h}^{n} \Gamma_{m l}^{h}\right) & \ldots & \sum_{l, m=1}^{n} g^{m l}\left(\frac{\partial \Gamma_{n l}^{n}}{\partial x_{m}}+\Gamma_{h l}^{n} \Gamma_{n m}^{h}-\Gamma_{n h}^{n} \Gamma_{m l}^{h}\right)
\end{array}\right] \\
& -(\mu+\lambda)\left[\begin{array}{ccc}
\sum_{l, m=1}^{n} g^{1 m} \frac{\partial \Gamma_{1 l}^{l}}{\partial x_{m}} & \cdots & \sum_{l, m=1}^{n} g^{1 m} \frac{\partial \Gamma_{n l}^{l}}{\partial x_{m}} \\
\vdots & & \vdots \\
\sum_{l, m=1}^{n} g^{n m} \frac{\partial \Gamma_{1 l}^{l}}{\partial x_{m}} & \cdots & \sum_{l, m=1}^{n} g^{n m} \frac{\partial \Gamma_{n l}^{l}}{\partial x_{m}}
\end{array}\right]-\mu\left[\begin{array}{ccc}
R_{1}^{1} & \cdots & R_{n}^{1} \\
\vdots & & \vdots \\
R_{1}^{n} & \cdots & R_{n}^{n}
\end{array}\right] .
\end{aligned}
$$

For each $\tau \in \mathbb{C}$, we denote

$$
\tau \mathbf{I}_{n}-\mathbf{A}_{g}=\mathbf{a}_{2}+\mathbf{a}_{1}+\mathbf{a}_{0},
$$

where

$$
\mathbf{a}_{2}(x, \xi):=\left(\tau-\mu \sum_{m, l=1}^{n} g^{m l} \xi_{m} \xi_{l}\right) \mathbf{I}_{n}-(\mu+\lambda)\left[\begin{array}{ccc}
\sum_{m=1}^{n} g^{1 m} \xi_{m} \xi_{1} & \cdots & \sum_{m=1}^{n} g^{1 m} \xi_{m} \xi_{n} \\
\vdots & & \vdots \\
\sum_{m=1}^{n} g^{n m} \xi_{m} \xi_{1} & \cdots & \sum_{m=1}^{n} g^{n m} \xi_{m} \xi_{n}
\end{array}\right],
$$




$$
\begin{aligned}
& \mathbf{a}_{1}(x, \xi):=-\mathrm{i} \mu\left(\sum_{m, l, s=1}^{n} g^{m l} \Gamma_{m l}^{s} \xi_{s}\right) \mathbf{I}_{n}+\mathrm{i} \mu\left[\begin{array}{ccc}
\sum_{m, l=1}^{n} 2 g^{m l} \Gamma_{1 m}^{1} \xi_{l} & \cdots & \sum_{m, l=1}^{n} 2 g^{m l} \Gamma_{n m}^{1} \xi_{l} \\
\vdots & & \vdots \\
\sum_{m, l=1}^{n} 2 g^{m l} \Gamma_{1 m}^{n} \xi_{l} & \cdots & \sum_{m, l=1}^{n} 2 g^{m l} \Gamma_{n m}^{n} \xi_{l}
\end{array}\right] \\
& +\mathrm{i}(\mu+\lambda)\left[\begin{array}{ccc}
\sum_{m, l=1}^{n} g^{1 m} \Gamma_{1 l}^{l} \xi_{m} & \cdots & \sum_{m, l=1}^{n} g^{1 m} \Gamma_{n l}^{l} \xi_{m} \\
\vdots & & \vdots \\
\sum_{m, l=1}^{n} g^{n m} \Gamma_{1 l}^{l} \xi_{m} & \cdots & \sum_{m, l=1}^{n} g^{n m} \Gamma_{n l}^{l} \xi_{m}
\end{array}\right] \\
& \mathbf{a}_{0}(x, \xi):=\mu\left[\begin{array}{ccc}
\sum_{l, m=1}^{n} g^{m l}\left(\frac{\partial \Gamma_{1 l}^{1}}{\partial x_{m}}+\Gamma_{h l}^{1} \Gamma_{1 m}^{h}-\Gamma_{1 h}^{1} \Gamma_{m l}^{h}\right) & \ldots & \sum_{l, m=1}^{n} g^{m l}\left(\frac{\partial \Gamma_{n l}^{1}}{\partial x_{m}}+\Gamma_{h l}^{1} \Gamma_{n m}^{h}-\Gamma_{n h}^{1} \Gamma_{m l}^{h}\right) \\
\vdots & & \vdots \\
\sum_{l, m=1}^{n} g^{m l}\left(\frac{\partial \Gamma_{l}^{n}}{\partial x_{m}}+\Gamma_{h l}^{n} \Gamma_{1 m}^{h}-\Gamma_{1 h}^{n} \Gamma_{m l}^{h}\right) & \ldots & \sum_{l, m=1}^{n} g^{m l}\left(\frac{\partial \Gamma_{n l}^{n}}{\partial x_{m}}+\Gamma_{h l}^{n} \Gamma_{n m}^{h}-\Gamma_{n h}^{n} \Gamma_{m l}^{h}\right)
\end{array}\right] \\
& +(\mu+\lambda)\left[\begin{array}{ccc}
\sum_{l, m=1}^{n} g^{1 m} \frac{\partial \Gamma_{1 l}^{l}}{\partial x_{m}} & \cdots & \sum_{l, m=1}^{n} g^{1 m} \frac{\partial \Gamma_{n l}^{l}}{\partial x_{m}} \\
\vdots & & \vdots \\
\sum_{l, m=1}^{n} g^{n m} \frac{\partial \Gamma_{1 l}^{l}}{\partial x_{m}} & \cdots & \sum_{l, m=1}^{n} g^{n m} \frac{\partial \Gamma_{n l}^{l}}{\partial x_{m}}
\end{array}\right]+\mu\left[\begin{array}{ccc}
R_{1}^{1} & \cdots & R_{n}^{1} \\
\vdots & & \vdots \\
R_{1}^{n} & \cdots & R_{n}^{n}
\end{array}\right] .
\end{aligned}
$$

We will calculate the full symbol of the resolvent operator $\left(\tau I-P_{g}\right)^{-1}$. Let $Q$ be a pseudodifferential operator which approximates the resolvent operator $\left(\tau I-P_{g}\right)^{-1}$, i.e.,

$$
\begin{aligned}
& \left(\tau I-P_{g}\right) Q=I \quad \bmod O P S^{-\infty} \\
& Q\left(\tau I-P_{g}\right)=I \bmod O P S^{-\infty} .
\end{aligned}
$$

Let

$$
\mathbf{q}(x, \xi, \tau) \sim \mathbf{q}_{-2}(x, \xi, \tau)+\mathbf{q}_{-3}(x, \xi, \tau)+\cdots+\mathbf{q}_{-2-l}(x, \xi, \tau)+\cdots
$$

be the expansion of the full symbol of $Q$. Suppose that the complex parameter $\tau$ have homogeneity 2 (This point of view stems from [68] or [27]). Let $\mathbf{q}_{-2-l}(x, \xi, \tau)$ be homogeneous of order $-2-l$ in the variables $\left(\xi, \tau^{1 / 2}\right)$. This infinite sum defines $\mathbf{q}(x, \xi, \tau)$ asymptotically. Our purpose is to determine $\mathbf{q}(x, \xi, \tau)$ so that

$$
\iota\left(\left(\tau I-P_{g}\right) Q\right) \sim \mathbf{I}_{n}
$$

where $\iota(T)$ denotes the full symbol of pseudodifferential operator $T$. By symbol formula (2.17) of the product of pseudodifferential operators, we can decompose the left-hand side of (3.7) into a sum of orders of homogeneity

$$
\sum_{\alpha \geq 0}\left(\partial_{\xi}^{\alpha}\left(\iota\left(\tau I-P_{g}\right)\right)\right) \cdot\left(D_{x}^{\alpha} \mathbf{q}\right) / \alpha ! \sim \mathbf{I}_{n}
$$

where $\partial_{\xi}^{\alpha}:=\frac{\partial^{|\alpha|}}{\partial \xi^{\alpha}}$. Noticing that $\iota\left(\tau I-P_{g}\right)=\left(\tau \mathbf{I}-\mathbf{A}_{g}\right)=\mathbf{a}_{2}+\mathbf{a}_{1}+\mathbf{a}_{0}$, we find by (3.8) that

$$
\iota\left(\left(\tau I-P_{g}\right) Q\right) \sim \sum_{l=0}^{\infty}\left(\sum_{l=j+|\alpha|+2-k}\left(\partial_{\xi}^{\alpha} \mathbf{a}_{k}\right) \cdot\left(D_{x}^{\alpha} \mathbf{q}_{-2-j}\right) / \alpha !\right) .
$$

The sum is over terms which are homogeneous of order $-l$. Thus (3.7) leads to the following equations

$$
\begin{aligned}
\mathbf{I}_{n} & =\sum_{0=j+|\alpha|+2-k}\left(\partial_{\xi}^{\alpha} \mathbf{a}_{k}\right)\left(D_{x}^{\alpha} \mathbf{q}_{-2-j}\right) / \alpha !=\mathbf{a}_{2} \mathbf{q}_{-2}, \\
0 & =\sum_{l=j+|\alpha|+2-k}\left(\partial_{\xi}^{\alpha} \mathbf{a}_{k}\right)\left(D_{x}^{\alpha} \mathbf{q}_{-2-j}\right) / \alpha !
\end{aligned}
$$




$$
=\mathbf{a}_{2} \mathbf{q}_{-2-l}+\sum_{\substack{l=j+|\alpha|+2-k \\ j<l}}\left(\partial_{\xi}^{\alpha} \mathbf{a}_{k}\right)\left(D_{x}^{\alpha} \mathbf{q}_{-2-j}\right) / \alpha !, \quad l \geq 1 .
$$

These equations determine the $\mathbf{q}_{-2-l}$ inductively. In other words, we have

$$
\mathbf{q}_{-2}=\mathbf{a}_{2}^{-1}, \text { and } \mathbf{q}_{-2-l}=-\mathbf{a}_{2}^{-1}\left(\sum_{j<l}\left(\partial_{\xi}^{\alpha} \mathbf{a}_{k}\right)\left(D_{x}^{\alpha} \mathbf{q}_{-2-j}\right) / \alpha !\right) \text { for } l=j+|\alpha|+2-k \geq 1 .
$$

In order to calculate the $\mathbf{a}_{2}^{-1}$, by a direct calculation we find that

$$
\begin{gathered}
{\left[\begin{array}{ccc}
\sum_{r=1}^{n} g^{1 r} \xi_{r} \xi_{1} & \cdots & \sum_{r=1}^{n} g^{1 r} \xi_{r} \xi_{n} \\
\vdots & & \vdots \\
\sum_{r=1}^{n} g^{n r} \xi_{r} \xi_{1} & \cdots & \sum_{r=1}^{n} g^{n r} \xi_{r} \xi_{n}
\end{array}\right]\left[\begin{array}{cccc}
\sum_{m=1}^{n} g^{1 m} \xi_{m} \xi_{1} & \cdots & \sum_{=1}^{n} g^{1 m} \xi_{m} \xi_{n} \\
\vdots & & & \vdots \\
\sum_{m=1}^{n} g^{n m} \xi_{m} \xi_{1} & \cdots & \sum_{m=1}^{n} g^{n m} \xi_{m} \xi_{n}
\end{array}\right]} \\
=\left(\begin{array}{llll}
\sum_{l, m=1}^{n} g^{l m} \xi_{l} \xi_{m}
\end{array}\right)\left[\begin{array}{ccc}
\sum_{r=1}^{n} g^{1 r} \xi_{r} \xi_{1} & \cdots & \sum_{r=1}^{n} g^{1 r} \xi_{r} \xi_{n} \\
\vdots & & \vdots \\
\sum_{r=1}^{n} g^{n r} \xi_{r} \xi_{1} & \cdots & \sum_{r=1}^{n} g^{n r} \xi_{r} \xi_{n}
\end{array}\right] .
\end{gathered}
$$

Thus, the following two matrices play a key role:

$$
F:=\left\{\mathbf{I}_{n},\left[\begin{array}{ccc}
\sum_{r=1}^{n} g^{1 r} \xi_{r} \xi_{1} & \cdots & \sum_{r=1}^{n} g^{1 r} \xi_{r} \xi_{n} \\
\vdots & & \vdots \\
\sum_{r=1}^{n} g^{n r} \xi_{r} \xi_{1} & \cdots & \sum_{r=1}^{n} g^{n r} \xi_{r} \xi_{n}
\end{array}\right]\right\} .
$$

The set $F$ of above two matrices can generate a matrix ring $\mathfrak{F}$ according to the usual matrix addition and multiplication of $\mathfrak{F}$ on the ring of functions. This implies that $\mathbf{a}_{2}^{-1}$ must have the following form:

$$
\mathbf{a}_{2}^{-1}=s_{1} \mathbf{I}_{n}+s_{2}\left[\begin{array}{ccc}
\sum_{r=1}^{n} g^{1 r} \xi_{r} \xi_{1} & \cdots & \sum_{r=1}^{n} g^{1 r} \xi_{r} \xi_{n} \\
\vdots & & \vdots \\
\sum_{r=1}^{n} g^{n r} \xi_{r} \xi_{1} & \cdots & \sum_{r=1}^{n} g^{n r} \xi_{r} \xi_{n}
\end{array}\right],
$$

where $s_{1}$ and $s_{2}$ are unknown functions which will be determined later. This key idea is inspired by Galois group theory for solving the polynomial equation (see [3] or [22]). Substituting (3.11) into $\mathbf{a}_{2} \mathbf{a}_{2}^{-1}=\mathbf{I}_{n}$, we have

$$
\begin{gathered}
\left\{\left(\tau-\mu \sum_{m, l=1}^{n} g^{m l} \xi_{m} \xi_{l}\right) \mathbf{I}_{n}-(\mu+\lambda)\left[\begin{array}{ccc}
\sum_{m=1}^{n} g^{1 m} \xi_{m} \xi_{1} & \cdots & \sum_{m=1}^{n} g^{1 m} \xi_{m} \xi_{n} \\
\vdots & & \vdots \\
\sum_{m=1}^{n} g^{n m} \xi_{m} \xi_{1} & \cdots & \sum_{m=1}^{n} g^{n m} \xi_{m} \xi_{n}
\end{array}\right]\right\}\left\{s_{1} \mathbf{I}_{n}\right. \\
\left.+s_{2}\left[\begin{array}{ccc}
\sum_{r=1}^{n} g^{1 r} \xi_{r} \xi_{1} & \cdots & \sum_{r=1}^{n} g^{1 r} \xi_{r} \xi_{n} \\
\vdots & & \vdots \\
\sum_{r=1}^{n} g^{n r} \xi_{r} \xi_{1} & \cdots & \sum_{r=1}^{n} g^{n r} \xi_{r} \xi_{n}
\end{array}\right]\right\}=\mathbf{I}_{n},
\end{gathered}
$$

i.e.,

$$
s_{1}\left(\tau-\mu \sum_{l, m=1}^{n} g^{l m} \xi_{l} \xi_{m}\right) \mathbf{I}_{n}+\left\{s_{2}\left(\tau-\mu \sum_{l, m=1}^{n} g^{l m} \xi_{l} \xi_{m}\right)-s_{1}(\mu+\lambda)\right.
$$




$$
\left.-s_{2}(\mu+\lambda) \sum_{l, m=1}^{n} g^{l m} \xi_{l} \xi_{m}\right\}\left[\begin{array}{ccc}
\sum_{r=1}^{n} g^{1 r} \xi_{r} \xi_{1} & \cdots & \sum_{r=1}^{n} g^{1 r} \xi_{r} \xi_{n} \\
\vdots & & \vdots \\
\sum_{r=1}^{n} g^{n r} \xi_{r} \xi_{1} & \cdots & \sum_{r=1}^{n} g^{n r} \xi_{r} \xi_{n}
\end{array}\right]=\mathbf{I}_{n} .
$$

Since the set $F$ is a basis of the matrix ring $\mathfrak{F}$, we get

$$
\left\{\begin{array}{l}
s_{1}\left(\tau-\mu \sum_{l, m=1}^{n} g^{l m} \xi_{l} \xi_{m}\right)=1, \\
s_{2}\left(\tau-\mu \sum_{l, m=1}^{n} g^{l m} \xi_{l} \xi_{m}\right)-s_{1}(\mu+\lambda)-s_{2}(\mu+\lambda) \sum_{l, m=1}^{n} g^{l m} \xi_{l} \xi_{m}=0 .
\end{array}\right.
$$

It follows that

$$
\left\{\begin{array}{l}
s_{1}=\frac{1}{\tau-\mu \sum_{l, m=1}^{n} g^{l m} \xi_{l} \xi_{m}}, \\
s_{2}=\frac{\mu+\lambda}{\left(\tau-\mu \sum_{l, m=1}^{n} g^{l m} \xi_{l} \xi_{m}\right)\left(\tau-(2 \mu+\lambda) \sum_{l, m=1}^{n} g^{l m} \xi_{l} \xi_{m}\right)}
\end{array}\right.
$$

Therefore

$$
\begin{aligned}
\mathbf{q}_{-2}= & \mathbf{a}_{2}^{-1}=\frac{1}{\tau-\mu \sum_{l, m=1}^{n} g^{l m} \xi_{l} \xi_{m}} \mathbf{I}_{n} \\
& +\frac{\mu+\lambda}{\left(\tau-\mu \sum_{l, m=1}^{n} g^{l m} \xi_{l} \xi_{m}\right)\left(\tau-(2 \mu+\lambda) \sum_{l, m=1}^{n} g^{l m} \xi_{l} \xi_{m}\right)}\left[\begin{array}{cccc}
\sum_{r=1}^{n} g^{1 r} \xi_{r} \xi_{1} & \ldots & \sum_{r=1}^{n} g^{1 r} \xi_{r} \xi_{n} \\
\vdots & & \vdots \\
\sum_{r=1}^{n} g^{n r} \xi_{r} \xi_{1} & \ldots & \sum_{r=1}^{n} g^{n r} \xi_{r} \xi_{n}
\end{array}\right] .
\end{aligned}
$$

Combining this and (3.10) we can get $\mathbf{q}_{-2-l}$ for all $l \geq 1$. For example, we can easily write out the first three terms $\mathbf{q}_{-2}, \mathbf{q}_{-3}, \mathbf{q}_{-4}$ :

$$
\begin{aligned}
& \mathbf{q}_{-2}(x, \xi, \tau)=\mathbf{a}_{2}^{-1} \\
& \mathbf{q}_{-3}(x, \xi, \tau)=-\mathbf{a}_{2}^{-1}\left(\mathbf{a}_{1} \mathbf{a}_{2}^{-1}-i \sum_{l=1}^{n} \frac{\partial \mathbf{a}_{2}}{\partial \xi_{l}} \frac{\partial \mathbf{a}_{2}^{-1}}{\partial x_{l}}\right) \\
& \mathbf{q}_{-4}(x, \xi, \tau)=-\mathbf{a}_{2}^{-1}\left(\sum_{j<2,|\alpha|=k-j}\left(\partial_{\xi}^{\alpha} \mathbf{a}_{k}\right)\left(D_{x}^{\alpha} \mathbf{q}_{-2-j}\right) / \alpha !\right) .
\end{aligned}
$$

From (3.13) we immediately have the following:

Lemma 3.1. Let $Q$ be a pseudodifferential operator satisfy (3.7) and let $\mathbf{q}_{-2}(x, \xi, \tau)$ be the principal symbol of $Q$. Then, for any $n \geq 1$,

$$
\begin{aligned}
& \mathbf{q}_{-2}(x, \xi, \tau)=\mathbf{a}_{2}^{-1}, \\
& \operatorname{Tr}\left(\mathbf{q}_{-2}(x, \xi, \tau)\right)=\frac{n}{\left(\tau-\mu \sum_{l, m=1}^{n} g^{l m} \xi_{l} \xi_{m}\right)}+\frac{(\mu+\lambda) \sum_{l, m=1}^{n} g^{l m} \xi_{l} \xi_{m}}{\left(\tau-\mu \sum_{l, m=1}^{n} g^{l m} \xi_{l} \xi_{m}\right)\left(\tau-(2 \mu+\lambda) \sum_{l, m=1}^{n} g^{l m} \xi_{l} \xi_{m}\right)},
\end{aligned}
$$

where $\mathbf{a}_{2}^{-1}$ is given by (3.13).

\section{Asymptotic expansion of trace of the integral Kernel}

Proof of Theorem 1.1. From the theory of elliptic operators (see [62, 663] [64], [65], [56], [72]), we see that the Navier-Lamé operator $-P_{g}$ can generate strongly continuous semigroups $\left(e^{-t P_{g}^{\mp}}\right)_{t \geq 0}$ with respect to the 
Dirichlet and Neumann boundary conditions, respectively, in suitable spaces of vector-valued functions (for example, in $\left[C_{0}(\Omega)\right]^{n}$ (see [72]) or in $\left[L^{2}(\Omega)\right]^{n}$ (see [16])). Furthermore, there exist matrix-valued functions $\mathbf{K}^{\mp}(t, x, y)$, which are called the integral kernels, such that (see [16] or p. 4 of [25])

$$
e^{-t P_{g}^{\mp}} \mathbf{w}_{0}(x)=\int_{\Omega} \mathbf{K}^{\mp}(t, x, y) \mathbf{w}_{0}(y) d y, \quad \mathbf{w}_{0} \in\left[L^{2}(\Omega)\right]^{n} .
$$

Let $\left\{u_{k}^{\mp}\right\}_{k=1}^{\infty}$ be the orthnormal eigenvectors of the elastic operators $P_{g}^{\mp}$ corresponding to the eigenvalues $\left\{\tau_{k}^{\mp}\right\}_{k=1}^{\infty}$, then the integral kernels $\mathbf{K}^{\mp}(t, x, y)=e^{-t P_{g}^{\mp}} \delta(x-y)$ are given by

$$
\mathbf{K}^{\mp}(t, x, y)=\sum_{k=1}^{\infty} e^{-t \tau_{k}^{\mp}} u_{k}^{\mp}(x) \otimes u_{k}^{\mp}(y) .
$$

This implies that the integrals of the traces of $\mathbf{K}^{\mp}(t, x, y)$ are actually spectral invariants:

$$
\int_{\Omega} \operatorname{Tr}\left(\mathbf{K}^{\mp}(t, x, x)\right) d V=\sum_{k=1}^{\infty} e^{-t \tau_{k}^{\mp}}
$$

We will combine calculus of symbols (see [69]) and "method of images" to deal with asymptotic expansions for the integrals of traces of integral kernels. Let $\mathcal{M}=\Omega \cup(\partial \Omega) \cup \Omega^{*}$ be the (closed) double of $\Omega$, and $\mathcal{P}$ the double to $\mathcal{M}$ of the operator $P_{g}$ on $\Omega$.

Let us explain the double Riemannian manifold $\mathcal{M}$ and the differential operator $\mathcal{P}$ more precisely, and introduce how to get them from the given Riemannian manifold $\Omega$ and the Navier-Lamé operator $P_{g}$. The double of $\Omega$ is the manifold $\Omega \cup_{\text {Id }} \Omega$, where Id : $\partial \Omega \rightarrow \partial \Omega$ is the identity map of $\partial \Omega$; it is obtained from $\Omega \sqcup \Omega$ by identifying each boundary point in one copy of $\Omega$ with same boundary point in the other. It is a smooth manifold without boundary, and contains two regular domains diffeomorphic to $\Omega$ (see, p. 226 of [48]). When considering the double differential system $\mathcal{P}$ crossing the boundary, we make use of the coordinates as follows. Let $x^{\prime}=\left(x_{1}, \cdots, x_{n-1}\right)$ be any local coordinates for $\partial \Omega$. For each point $\left(x^{\prime}, 0\right) \in \partial \Omega$, let $x_{n}$ denote the parameter along the unit-speed geodesic starting at $\left(x^{\prime}, 0\right)$ with initial direction given by the inward boundary normal to $\partial \Omega$ (Clearly, $x_{n}$ is the geodesic distance from the point $\left(x^{\prime}, 0\right)$ to the point $\left(x^{\prime}, x_{n}\right)$ ). In such coordinates $x_{n}>0$ in $\Omega$, and $\partial \Omega$ is locally characterized by $x_{n}=0$ (see, 49. or [75]). Since the Navier-Lamé operator is a linear differential operator defined on $\Omega$, it can be further denoted as $P_{g}:=P\left(g^{\alpha \beta}(x), g^{\alpha n}(x), g^{n \beta}(x), g^{n n}(x), \frac{\partial}{\partial x_{1}}, \cdots, \frac{\partial}{\partial x_{n-1}}, \frac{\partial}{\partial x_{n}}\right)$, where $1 \leq \alpha, \beta \leq n-1$. Let $\varsigma:\left(x_{1}, \cdots, x_{n-1}, x_{n}\right) \mapsto\left(x_{1}, \cdots, x_{n-1},-x_{n}\right)$ be the reflection with respect to the boundary $\partial \Omega$ in $\mathcal{M}$ (here we always assume $x_{n} \geq 0$ ). Then we can get the $\Omega^{*}$ from the given $\Omega$ and $\varsigma$. Now, we discuss the change of the metric $g$ from $\Omega$ to $\Omega^{*}$ by $\varsigma$. Recall that the Riemannian metric $\left(g_{i j}\right)$ is given in the local coordinates $x_{1}, \cdots, x_{n}$, i.e., $g_{i j}\left(x_{1}, \cdots, x_{n}\right)$. In terms of the new coordinates $z_{1}, \cdots, z_{n}$, with $x_{i}=x_{i}\left(z_{1}, \cdots, z_{n}\right), i=$ $1, \cdots, n$, the same metric is given by the functions $\tilde{g}_{i j}=\tilde{g}_{i j}\left(z_{1}, \cdots, z_{n}\right)$, where

$$
\tilde{g}_{i j}=\frac{\partial x_{k}}{\partial z_{i}} g_{k l} \frac{\partial x_{l}}{\partial z_{j}}
$$

If $\varsigma$ is a coordinate change in a neighborhood intersecting with $\partial \Omega$

$$
\left\{\begin{array}{l}
x_{1}=z_{1}, \\
\cdots \cdots \\
x_{n-1}=z_{n-1}, \\
x_{n}=-z_{n},
\end{array}\right.
$$

then its Jacobian matrix is

$$
J:=\left(\begin{array}{cccc}
\frac{\partial x_{1}}{\partial z_{1}} & \cdots & \frac{\partial x_{1}}{\partial z_{n-1}} & \frac{\partial x_{1}}{\partial z_{n}} \\
\vdots & \ddots & \vdots & \vdots \\
\frac{\partial x_{n-1}}{\partial z_{1}} & \cdots & \frac{\partial x_{n-1}}{\partial z_{n-1}} & \frac{\partial x_{n-1}}{\partial z_{n}} \\
\frac{\partial x_{n}}{\partial z_{1}} & \cdots & \frac{\partial x_{n}}{\partial z_{n-1}} & \frac{\partial x_{n}}{\partial z_{n}}
\end{array}\right)=\left(\begin{array}{cccc}
1 & \cdots & 0 & 0 \\
\vdots & \ddots & \vdots & \vdots \\
0 & \cdots & 1 & 0 \\
0 & \cdots & 0 & -1
\end{array}\right) .
$$

Using this and (4.3), we immediately obtain the corresponding metric on the $\Omega^{*}$ : (see [52, 58] or p. 10169, p. 10183 and p. 10187 of [55] )

$$
g_{j k}(\stackrel{*}{x})=-g_{j k}(x) \quad \text { for } j<k=n \text { or } k<j=n,
$$




$$
\begin{aligned}
g(*) & =g_{j k}(x) \quad \text { for } j, k<n \text { or } j=k=n, \\
g_{j k}(x) & =0 \text { for } j<k=n \text { or } k<j=n \text { on } \partial \Omega,
\end{aligned}
$$

where $x_{n}(\stackrel{*}{x})=-x_{n}(x)$. We denote such a new (isometric) metric on $\Omega^{*}$ as $g^{*}$. It is easy to verify that

$$
\left[\begin{array}{cccc}
g_{11}(x) & \cdots & g_{1, n-1}(x) & -g_{1 n}(x) \\
\vdots & \ddots & \vdots & \vdots \\
g_{n-1,1}(x) & \cdots & g_{n-1, n-1}(x) & -g_{n-1, n}(x) \\
-g_{n 1}(x) & \cdots & -g_{n, n-1}(x) & g_{n n}(x)
\end{array}\right]^{-1}=\left[\begin{array}{cccc}
g^{11}(x) & \cdots & g^{1, n-1}(x) & -g^{1 n}(x) \\
\vdots & \ddots & \vdots & \vdots \\
g^{n-1,1}(x) & \cdots & g^{n-1, n-1}(x) & -g^{n-1, n}(x) \\
-g^{n 1}(x) & \cdots & -g^{n, n-1}(x) & g^{n n}(x)
\end{array}\right]
$$

where $\left[g^{j k}(x)\right]_{n \times n}$ is the inverse of $\left[g_{j k}(x)\right]_{n \times n}$. In addition, by this reflection $\varsigma$, the differential operators $\frac{\partial}{\partial x_{1}}, \cdots, \frac{\partial}{\partial x_{n-1}}, \frac{\partial}{\partial x_{n}}\left(\right.$ defined on $\Omega$ ) are changed to $\frac{\partial}{\partial x_{1}}, \cdots, \frac{\partial}{\partial x_{n-1}},-\frac{\partial}{\partial x_{n}}\left(\right.$ defined on $\left.\Omega^{*}\right)$, respectively. We define

$$
\mathcal{P}= \begin{cases}P_{g} & \text { on } \Omega \\ P^{*} & \text { on } \Omega^{*}\end{cases}
$$

where

$$
P^{*}:=P\left(g^{\alpha \beta}(\stackrel{*}{x}),-g^{\alpha n}(\stackrel{*}{x}),-g^{n \beta}(\stackrel{*}{x}), g^{n n}(\stackrel{*}{x}), \frac{\partial}{\partial x_{1}}, \cdots, \frac{\partial}{\partial x_{n-1}},-\frac{\partial}{\partial x_{n}}\right),
$$

and $\stackrel{*}{x}=\left(x^{\prime},-x_{n}\right) \in \Omega^{*}$. Clearly, the differential operator $P^{*}$ is obtained by $P_{g}$ and the reflection $\varsigma$, that is, $P^{*}$ is got if we replace $g^{\alpha \beta}(x), g^{\alpha n}(x), g^{n \beta}(x), g^{n n}(x), \frac{\partial}{\partial x_{n}}$ by $g^{\alpha \beta}(\stackrel{*}{x}),-g^{\alpha n}(\stackrel{*}{x}),-g^{n \beta}(\stackrel{*}{x}), g^{n n}(\stackrel{*}{x}),-\frac{\partial}{\partial x_{n}}$ in $P_{g}=P\left(g^{\alpha \beta}(x), g^{\alpha n}(x), g^{n \beta}(x), g^{n n}(x), \frac{\partial}{\partial x_{1}}, \cdots, \frac{\partial}{\partial x_{n-1}}, \frac{\partial}{\partial x_{n}}\right)$, respectively. Note that $g^{\alpha \beta}(\stackrel{*}{x})=g^{\alpha \beta}(x)$, $-g^{\alpha n}(\stackrel{*}{x})=g^{\alpha n}(x),-g^{n \beta}(\stackrel{*}{x})=g^{n \beta}(x)$ and $g^{n n}(\stackrel{*}{x})=g^{n n}(x)$. In view of the metric matrices $g$ and $g^{*}$ have the same order principal minor determinants, we see that $\mathcal{P}$ is still a linear elliptic differential operator on $\mathcal{M}$.

Let $\mathbf{K}(t, x, y)$ be the fundamental solution of the parabolic system

$$
\begin{cases}\frac{\partial \mathbf{u}}{\partial t}+\mathcal{P} \mathbf{u}=0 & \text { in }(0,+\infty) \times \mathcal{M} \\ \mathbf{u}=\phi & \text { on }\{0\} \times \mathcal{M}\end{cases}
$$

That is, for any $t \geq 0$ and $x, y \in \mathcal{M}$,

$$
\begin{cases}\frac{\partial \mathbf{K}(t, x, y)}{\partial t}+\mathcal{P} \mathbf{K}(t, x, y)=0 & \text { for } t>0, x, y \in \mathcal{M} \\ \mathbf{K}(0, x, y)=\boldsymbol{\delta}(x-y) & \text { for } x, y \in \mathcal{M}\end{cases}
$$

Here the operator $\mathcal{P}$ is acted in the third argument $y$ of $\mathbf{K}(t, x, y)$.

Clearly, the coefficients occurring in $\mathcal{P}$ jump as $x$ crosses the $\partial \Omega$ (since the extended metric $g$ is $C^{0}$-smooth on whole $\mathcal{M}$ and $C^{\infty}$-smooth in $\left.\mathcal{M} \backslash \partial \Omega\right)$, but $\frac{\partial \mathbf{u}}{\partial t}+\mathcal{P} \mathbf{u}=0$ with $\mathbf{u}(0, x)=\phi(x)$ still has a nice fundamental solution $\mathbf{K}$ of class $C^{1}((0,+\infty) \times \mathcal{M} \times \mathcal{M}) \cap C^{\infty}((0,+\infty) \times(\mathcal{M} \backslash \partial \Omega) \times(\mathcal{M} \backslash \partial \Omega))$, approximable even on $\partial \Omega$ by Levi's sum (see [55], or another proof below). Now, let us restrict $x, y \in \Omega$. It can be verified that $\mathbf{K}^{-}(t, x, y):=\mathbf{K}(t, x, y)-\mathbf{K}(t, x, \stackrel{*}{y})$ and $\mathbf{K}^{+}:=\mathbf{K}(t, x, y)+\mathbf{K}(t, x, \stackrel{*}{y})$ are the Green functions of

$$
\begin{cases}\frac{\partial \mathbf{u}}{\partial t}+P_{g} \mathbf{u}=0 & \text { in }(0,+\infty) \times \Omega \\ \mathbf{u}=\phi & \text { on }\{0\} \times \Omega\end{cases}
$$

with zero Dirichlet and Neumann boundary conditions, respectively, where $y=\left(y^{\prime}, y_{n}\right), y_{n} \geq 0$, and $\stackrel{*}{y}:=$ $\varsigma\left(y^{\prime}, y_{n}\right)=\left(y^{\prime},-y_{n}\right)$. In other words,

$$
\left\{\begin{array}{l}
\frac{\partial \mathbf{K}^{-}(t, x, y)}{\partial t}+P_{g} \mathbf{K}^{-}(t, x, y)=0, \quad t>0, x, y \in \Omega \\
\mathbf{K}^{-}(t, x, y)=0, \quad t>0, x \in \Omega, y \in \partial \Omega \\
\mathbf{K}^{-}(0, x, y)=\boldsymbol{\delta}(x-y), \quad x, y \in \Omega
\end{array}\right.
$$

and

$$
\left\{\begin{array}{l}
\frac{\partial \mathbf{K}^{+}(t, x, y)}{\partial t}+P_{g} \mathbf{K}^{+}(t, x, y)=0, \quad t>0, x, y \in \Omega \\
\frac{\partial \mathbf{K}^{+}(t, x, y)}{\partial \boldsymbol{\nu}}=0, \quad t>0, x \in \Omega, \quad y \in \partial \Omega \\
\mathbf{K}^{+}(0, x, y)=\boldsymbol{\delta}(x-y), \quad x, y \in \Omega
\end{array}\right.
$$


where $\frac{\partial \mathbf{K}^{+}}{\partial \boldsymbol{\nu}}:=\mu\left(\nabla \mathbf{K}^{+}+\left(\nabla \mathbf{K}^{+}\right)^{T}\right) \boldsymbol{\nu}+\lambda\left(\operatorname{div} \mathbf{K}^{+}\right) \boldsymbol{\nu}$ on $\partial \Omega$. In fact, for any $t>0, x, y \in \Omega$, we have $P_{g} \mathbf{K}(t, x, y)=\mathcal{P} \mathbf{K}(t, x, y)$, so that

$$
\left\{\begin{array}{l}
\left(\frac{\partial}{\partial t}+P_{g}\right) \mathbf{K}(t, x, y)=\left(\frac{\partial}{\partial t}+\mathcal{P}\right) \mathbf{K}(t, x, y)=0, \\
\mathbf{K}(0, x, y)=\boldsymbol{\delta}(x-y)
\end{array}\right.
$$

by (4.11). Noting that the Jacobian matrix of the reflection $\varsigma$ is $J$ (see (4.5)), it follows from chain rule that for any fixed $t>0$ and $x \in \Omega$, and any $y=\left(y^{\prime}, y_{n}\right) \in \Omega$,

$$
\begin{aligned}
& {\left.\left[P_{g}(\mathbf{K}(t, x, \stackrel{*}{y}))\right]\right|_{\text {evaluated at the point } y}} \\
& =\left.\left[P_{g}\left(\mathbf{K}\left(t, x, \varsigma\left(y^{\prime}, y_{n}\right)\right)\right)\right]\right|_{\text {evaluated at the point }\left(y^{\prime}, y_{n}\right)} \\
& =\left.\left[P_{g}\left(\mathbf{K}\left(t, x,\left(y^{\prime},-y_{n}\right)\right)\right)\right]\right|_{\text {evaluated at the point }\left(y^{\prime}, y_{n}\right)} \\
& =\left.\left\{\left[P\left(g^{\alpha \beta}(y), g^{\alpha n}(y), g^{n \beta}(x), g^{n n}(x), \frac{\partial}{\partial y_{1}}, \cdots, \frac{\partial}{\partial y_{n-1}}, \frac{\partial}{\partial y_{n}}\right)\right] \mathbf{K}\left(t, x,\left(y^{\prime},-y_{n}\right)\right)\right\}\right|_{\text {evaluated at }\left(y^{\prime}, y_{n}\right)} \\
& =\left.\left\{\left[P\left(g^{\alpha \beta}(\stackrel{*}{y}),-g^{\alpha n}(\stackrel{*}{y}),-g^{n \beta}(\stackrel{*}{y}), g^{n n}(\stackrel{*}{y}), \frac{\partial}{\partial y_{1}}, \cdots, \frac{\partial}{\partial y_{n-1}},-\frac{\partial}{\partial y_{n}}\right)\right] \mathbf{K}(t, x, y)\right\}\right|_{\text {evaluated at }} \stackrel{*}{y}=\left(y^{\prime},-y_{n}\right) \\
& =\left.P^{*}(\mathbf{K}(t, x, \stackrel{*}{y}))\right|_{\text {evaluated at the point }} \stackrel{*}{y}=\left(y^{\prime},-y_{n}\right) \cdot
\end{aligned}
$$

That is, the action of $P_{g}$ to $\mathbf{K}(t, x, \stackrel{*}{y})$ at the point $y=\left(y^{\prime}, y_{n}\right)$ is just the action of $P^{*}$ to $\mathbf{K}(t, x, \stackrel{*}{y})$ at the point ${ }^{*}=\left(y^{\prime},-y_{n}\right)$. Because of $\varsigma\left(y^{\prime}, y_{n}\right)=\left(y^{\prime},-y_{n}\right) \in \Omega^{*}$, we see

$$
\left.P^{*}(\mathbf{K}(t, x, \stackrel{*}{y}))\right|_{\text {evaluated at the point } \stackrel{*}{y}=\left(y^{\prime},-y_{n}\right)}=\left.\mathcal{P}(\mathbf{K}(t, x, \stackrel{*}{y}))\right|_{\text {evaluated at the point }} \stackrel{*}{y}=\left(y^{\prime},-y_{n}\right) .
$$

For any $t>0, x \in \Omega$ and $\left(y^{\prime},-y_{n}\right) \in \Omega^{*}$, we have

$$
\left(\frac{\partial}{\partial t}+\mathcal{P}\right)\left(\mathbf{K}\left(t, x,\left(y^{\prime},-y_{n}\right)\right)\right)=0 .
$$

In addition, $\mathbf{K}\left(t, x,\left(y^{\prime},-y_{n}\right)\right)=\mathbf{K}(t, x, \varsigma(y))$ for any $t>0, x, y \in \Omega$. By virtue of $x \neq\left(y^{\prime},-y_{n}\right)$, this leads to $\mathbf{K}\left(0, x,\left(y^{\prime},-y_{n}\right)\right)=0$ and

$$
\left(\frac{\partial}{\partial t}+P_{g}\right)\left(\mathbf{K}\left(t, x,\left(y^{\prime},-y_{n}\right)\right)\right)=0 \text { for any } t>0, x \in \Omega \text { and }\left(y^{\prime},-y_{n}\right) \in \Omega^{*},
$$

i.e.,

$$
\left\{\begin{array}{l}
\left(\frac{\partial}{\partial t}+P_{g}\right) \mathbf{K}(t, x, \stackrel{*}{y})=0 \text { for any } t>0, x \in \Omega \text { and } \stackrel{*}{y} \in \Omega^{*}, \\
\mathbf{K}(0, x, \stackrel{*}{y})=0 \text { for any } x, y \in \Omega .
\end{array}\right.
$$

Combining (4.12) and (4.13), we obtain that

$$
\left\{\begin{array}{l}
\left(\frac{\partial}{\partial t}+P_{g}\right)(\mathbf{K}(t, x, y)-\mathbf{K}(t, x, \stackrel{*}{y}))=0 \text { for any } t>0, x, y \in \Omega \\
\mathbf{K}(0, x, y)-\mathbf{K}(0, x, \stackrel{*}{y})=\boldsymbol{\delta}(x-y) \text { for any } x, y \in \Omega
\end{array}\right.
$$

$\mathbf{K}(t, x, y)$ is $C^{1}$-smooth with respect to $y$ in $\mathcal{M}$ for any fixed $t>0$ and $x \in \Omega$, so does it on the hypersurface $\partial \Omega$. Therefore, we get that $\mathbf{K}^{-}(t, x, y)$ (respectively $\left.\mathbf{K}^{+}(t, x, y)\right)$ is the Green function in $\Omega$ with the Dirichlet (respectively, Neumann) boundary condition on $\partial \Omega$.

To show $C^{1}$-regularity of the fundamental solution $\mathbf{K}(t, x, y)$, it suffices to prove $C_{l o c}^{1,1}$-regularity for the solution $\mathbf{u}$ of the elliptic system $\mathcal{P} \mathbf{u}=\mathbf{f}$ in $\mathcal{M}$. This immediately follows from Xiong's result [78] of $C_{\text {loc }}^{1,1}$ regularity for solution of elliptic system

$$
\left\{\begin{array}{l}
L \mathbf{u}=\mathbf{f} \text { in } U \\
\mathbf{u}=\phi \text { on } \partial U
\end{array}\right.
$$


with piecewise uniformly Hölder continuous coefficients and $\mathbf{f}$ on both sides of a general $(n-1)$-dimensional embedded $C^{1, \alpha}$ hypersurface $S$ (the coefficients might be discontinuous cross this hypersurface), where $L$ is a general elliptic operator of second order, $U$ is a bounded domain in $\mathbb{R}^{n}$ with smooth boundary, $\phi \in[C(\partial U)]^{n}$ and $S \cap U \neq \emptyset$. This result (see, Theorem 1.2 of [78]) can also be applied to our case for $C_{l o c}^{1,1}$-regularity of the fundamental solution in an $n$-dimensional Riemannian manifold (see [55]). When the coefficients of an elliptic (or parabolic) system are piecewise smooth between an $(n-1)$-dimensional hyperplane (might be discontinuous cross this hyperplane), the corresponding $C^{1+\alpha / 2, \alpha}$-regularity for solution of an elliptic system was obtained by Dong (see Remark 5 of p. 141 in [20]).

Therefore, the integral kernels $\mathbf{K}^{\mp}(t, x, y)$ of $\frac{\partial \mathbf{u}}{\partial t}+P_{g}^{\mp} \mathbf{u}=0$ can be expressed on $(0, \infty) \times \Omega \times \Omega$ as

$$
\mathbf{K}^{\mp}(t, x, y)=\mathbf{K}(t, x, y) \mp \mathbf{K}(t, x, \stackrel{*}{y}),
$$

$\stackrel{*}{y}$ being the double of $y \in \Omega$ (see, p. 53 of $[60]$ ). Since the strongly continuous semigroup $\left(e^{-t \mathcal{P}}\right)_{t \geq 0}$ can also be represented as

$$
e^{-t \mathcal{P}}=\frac{1}{2 \pi i} \int_{\mathcal{C}} e^{-t \tau}(\tau I-\mathcal{P})^{-1} d \tau,
$$

where $\mathcal{C}$ is a suitable curve in the complex plane in the positive direction around the spectrum of $\mathcal{P}$ (i.e., a contour around the positive real axis). It follows that

$$
\mathbf{K}(t, x, y)=e^{-t \mathcal{P}} \delta(x-y)=\frac{1}{(2 \pi)^{n}} \int_{\mathbb{R}^{n}} e^{i(x-y) \cdot \xi}\left(\frac{1}{2 \pi i} \int_{\mathcal{C}} e^{-t \tau} \iota\left((\tau I-\mathcal{P})^{-1}\right) d \tau\right) d \xi, \quad \forall x, y \in \mathcal{M}
$$

We claim that

$$
\frac{1}{2 \pi i} \int_{\mathcal{C}}(\tau I-\mathcal{P})^{-1} e^{-t \tau} \delta(x-y) d \tau=\frac{1}{2 \pi i} \int_{\mathcal{C}}\left(\int_{\mathbb{R}^{n}} e^{i(x-y) \cdot \xi} \sum_{j \leq-2} \mathbf{q}_{j}(x, \xi, \tau) d \xi\right) e^{-t \tau} d \tau .
$$

In fact, for any smooth vector-valued function $\phi$ with compact we have

$$
\begin{aligned}
\left(e^{-t \mathcal{P}} \phi\right)(x) & =\left(\frac{1}{2 \pi i} \int_{\mathcal{C}} e^{-t \tau}(\tau I-\mathcal{P})^{-1} d \tau\right) \phi(x) \\
& =\frac{1}{2 \pi i} \int_{\mathcal{C}} e^{-t \tau}\left(\int_{\mathbb{R}^{n}} e^{i x \cdot \xi} \sum_{j \leq-2} \mathbf{q}_{j}(x, \xi, \tau) \hat{\boldsymbol{\phi}}(\xi) d \xi\right) d \tau .
\end{aligned}
$$

On the one hand, from the left-hand side of (4.17), we get

$$
\begin{aligned}
\int\left[\left(\frac{1}{2 \pi i} \int_{\mathcal{C}}(\tau I-\mathcal{P})^{-1} e^{-t \tau} d \tau\right)(\delta(x-y))\right] \phi(y) d y \\
\quad=\left(\frac{1}{2 \pi i} \int_{\mathcal{C}}(\tau-\mathcal{P})^{-1} e^{-t \tau} d \tau\right) \phi(x)=e^{-t \mathcal{P}} \phi(x) .
\end{aligned}
$$

On the other hand, from the right-hand side of (4.17) we obtain

$$
\begin{aligned}
\int\left[\frac{1}{2 \pi i}\right. & \left.\int_{C}\left(\int_{\mathbb{R}^{n}} e^{i(x-y) \cdot \xi} \sum_{j \leq-2} \mathbf{q}_{j}(x, \xi, \tau) d \xi\right) e^{-t \tau} d \tau\right] \boldsymbol{\phi}(y) d y \\
& =\frac{1}{2 \pi i} \int_{C}\left(\int_{\mathbb{R}^{n}} e^{i x \cdot \xi} \sum_{j \leq-2} \mathbf{q}_{j}(x, \xi, \tau) d \xi\right) e^{-t \tau} d \tau \int e^{-y \cdot \xi} \boldsymbol{\phi}(y) d y \\
& =\frac{1}{2 \pi i} \int_{C}\left(\int_{\mathbb{R}^{n}} e^{i x \cdot \xi} \sum_{j \leq-2} \mathbf{q}_{j}(x, \xi, \tau) \hat{\boldsymbol{\phi}}(\xi) d \xi\right) e^{-t \tau} d \tau=e^{-t \mathcal{P}} \boldsymbol{\phi}(x) .
\end{aligned}
$$

Thus, the desired identity (4.17) is asserted by (4.18) and (4.19).

In particular, for every $x \in \Omega$,

$$
\begin{aligned}
\mathbf{K}(t, x, x) & =e^{-t \mathcal{P}} \delta(x-x)=\frac{1}{(2 \pi)^{n}} \int_{\mathbb{R}^{n}}\left(\frac{1}{2 \pi i} \int_{\mathcal{C}} e^{-t \tau} \iota\left((\tau I-\mathcal{P})^{-1}\right) d \tau\right) d \xi \\
& =\frac{1}{(2 \pi)^{n}} \int_{\mathbb{R}^{n}}\left(\frac{1}{2 \pi i} \int_{\mathcal{C}} e^{-t \tau} \iota\left(\left(\tau I-P_{g}\right)^{-1}\right) d \tau\right) d \xi
\end{aligned}
$$




$$
\begin{aligned}
& =\frac{1}{(2 \pi)^{n}} \int_{\mathbb{R}^{n}}\left(\frac{1}{2 \pi i} \int_{\mathcal{C}} e^{-t \tau} \sum_{l \geq 0} q_{-2-l}(x, \xi, \tau) d \tau\right) d \xi, \\
\mathbf{K}(t, x, *) & =e^{-t \mathcal{P}} \delta(x-\stackrel{*}{x})=\frac{1}{(2 \pi)^{n}} \int_{\mathbb{R}^{n}} e^{i(x-x) \cdot \xi}\left(\frac{1}{2 \pi i} \int_{\mathcal{C}} e^{-t \tau} \iota\left((\tau I-\mathcal{P})^{-1}\right) d \tau\right) d \xi \\
& =\frac{1}{(2 \pi)^{n}} \int_{\mathbb{R}^{n}} e^{i(x-*) \cdot \xi}\left(\frac{1}{2 \pi i} \int_{\mathcal{C}} e^{-t \tau} \iota\left((\tau I-\mathcal{P})^{-1}\right) d \tau\right) d \xi \\
& =\frac{1}{(2 \pi)^{n}} \int_{\mathbb{R}^{n}} e^{i(x-*) \cdot \xi}\left(\frac{1}{2 \pi i} \int_{\mathcal{C}} e^{-t \tau} \sum_{l \geq 0} q_{-2-l}(x, \xi, \tau) d \tau\right) d \xi,
\end{aligned}
$$

where $\sum_{l>0} \mathbf{q}_{-2-l}(x, \xi, \tau)$ is the full symbol of $\left(\tau I-P_{g}\right)^{-1}$.

Firstly, from the discussion of previous section, we know that

$$
\begin{aligned}
& \mathbf{q}_{-2}(x, \xi, \tau)=\frac{1}{\tau-\mu \sum_{l, m=1}^{n} g^{l m} \xi_{l} \xi_{m}} \mathbf{I}_{n} \\
& +\frac{\mu+\lambda}{\left(\tau-\mu \sum_{l, m=1}^{n} g^{l m} \xi_{l} \xi_{m}\right)\left(\tau-(2 \mu+\lambda) \sum_{l, m=1}^{n} g^{l m} \xi_{l} \xi_{m}\right)}\left[\begin{array}{cccc}
\sum_{r=1}^{n} g^{1 r} \xi_{r} \xi_{1} & \ldots & \sum_{r=1}^{n} g^{1 r} \xi_{r} \xi_{n} \\
\vdots & & \vdots \\
\sum_{r=1}^{n} g^{n r} \xi_{r} \xi_{1} & \ldots & \sum_{r=1}^{n} g^{n r} \xi_{r} \xi_{n}
\end{array}\right]
\end{aligned}
$$

and

$$
\operatorname{Tr}\left(\mathbf{q}_{-2}(x, \xi, \tau)\right)=\frac{n}{\left(\tau-\mu \sum_{l, m=1}^{n} g^{l m} \xi_{l} \xi_{m}\right)}+\frac{(\mu+\lambda) \sum_{l, m=1}^{n} g^{l m} \xi_{l} \xi_{m}}{\left(\tau-\mu \sum_{l, m=1}^{n} g^{l m} \xi_{l} \xi_{m}\right)\left(\tau-(2 \mu+\lambda) \sum_{l, m=1}^{n} g^{l m} \xi_{l} \xi_{m}\right)} .
$$

For each $x \in \Omega$, we use a geodesic normal coordinate system centered at this $x$. It follows from $\S 11$ of Chap. 1 in [74] that in such a coordinate system, $g_{j k}(x)=\delta_{j k}$ and $\Gamma_{j k}^{l}(x)=0$. Then (4.23) reduces to

$$
\operatorname{Tr}\left(\mathbf{q}_{-2}(x, \xi, \tau)\right)=\frac{n}{\left(\tau-\mu|\xi|^{2}\right)}+\frac{(\mu+\lambda)|\xi|^{2}}{\left(\tau-\mu|\xi|^{2}\right)\left(\tau-(2 \mu+\lambda)|\xi|^{2}\right)},
$$

where $|\xi|=\sqrt{\sum_{k=1}^{n} \xi_{k}^{2}}$ for any $\xi \in \mathbb{R}^{n}$. By applying the residue theorem (see, for example, Chap. 4 , 55 in [2]) we get

$$
\frac{1}{2 \pi i} \int_{\mathcal{C}} e^{-t \tau}\left(\frac{n}{\left(\tau-\mu|\xi|^{2}\right)}+\frac{(\mu+\lambda)|\xi|^{2}}{\left(\tau-\mu|\xi|^{2}\right)\left(\tau-(2 \mu+\lambda)|\xi|^{2}\right)}\right) d \tau=(n-1) e^{-t \mu|\xi|^{2}}+e^{-t(2 \mu+\lambda)|\xi|^{2}} .
$$

It follows that

$$
\begin{aligned}
\frac{1}{(2 \pi)^{n}} & \int_{\mathbb{R}^{n}}\left(\frac{1}{2 \pi i} \int_{\mathcal{C}} e^{-t \tau} \operatorname{Tr}\left(\mathbf{q}_{-2}(x, \xi, \tau)\right) d \tau\right) d \xi \\
& =\frac{1}{(2 \pi)^{n}} \int_{\mathbb{R}^{n}}\left((n-1) e^{-t \mu|\xi|^{2}}+e^{-t(2 \mu+\lambda)|\xi|^{2}}\right) d \xi \\
& =\frac{n-1}{(4 \pi \mu t)^{n / 2}}+\frac{1}{(4 \pi(2 \mu+\lambda) t)^{n / 2}},
\end{aligned}
$$

and hence

$$
\int_{\Omega}\left\{\frac{1}{(2 \pi)^{n}} \int_{\mathbb{R}^{n}}\left(\frac{1}{2 \pi i} \int_{\mathcal{C}} e^{-t \tau} \operatorname{Tr}\left(\mathbf{q}_{-2}(x, \xi, \tau)\right) d \tau\right) d \xi\right\} d V=\left(\frac{n-1}{(4 \pi \mu t)^{n / 2}}+\frac{1}{(4 \pi(2 \mu+\lambda) t)^{n / 2}}\right) \operatorname{Vol}(\Omega) .
$$

In the above discussion, if we replace $x \in \Omega$ by ${ }^{*} \in \Omega^{*}$, then (4.22) will become

$$
\mathbf{q}_{-2}(\stackrel{*}{x}, \xi, \tau)=\frac{1}{\tau-\mu \sum_{l, m=1}^{n}\left(g^{l m}(\stackrel{*}{x})\right) \xi_{l} \xi_{m}} \mathbf{I}_{n}+\frac{\mu+\lambda}{\left(\tau-\mu \sum_{l, m=1}^{n}\left(g^{l m}(\stackrel{*}{x})\right) \xi_{l} \xi_{m}\right)\left(\tau-(2 \mu+\lambda) \sum_{l, m=1}^{n}\left(g^{l m}(\stackrel{*}{x})\right) \xi_{l} \xi_{m}\right)}
$$




$$
\times\left[\begin{array}{cccc}
\sum_{r=1}^{n}\left(g^{1 r}\left(*^{*}\right)\right) \xi_{r} \xi_{1} & \cdots & \sum_{r=1}^{n}\left(g^{1 r}\left({ }^{*}\right)\right) \xi_{r} \xi_{n-1} & \sum_{r=1}^{n}\left(-g^{1 r}\left({ }^{*}\right)\right) \xi_{r} \xi_{n} \\
\vdots & & \vdots & \\
\sum_{r=1}^{n}\left(g^{n-1, r}\left({ }^{*}\right)\right) \xi_{r} \xi_{1} & \cdots & \sum_{r=1}^{n}\left(g^{n-1, r}\left(\stackrel{*}{x}^{*}\right)\right) \xi_{r} \xi_{n-1} & \sum_{r=1}^{n}\left(-g^{n-1, r}\left({ }^{*}\right)\right) \xi_{r} \xi_{n} \\
\sum_{r=1}^{n}\left(-g^{n r}\left({ }^{*}\right)\right) \xi_{r} \xi_{1} & \cdots & \sum_{r=1}^{n}\left(-g^{n r}\left({ }^{*}\right)\right) \xi_{r} \xi_{n-1} & \sum_{r=1}^{n}\left(g^{n r}(*)\right) \xi_{r} \xi_{n}
\end{array}\right]
$$

and

$$
\operatorname{Tr}\left(\mathbf{q}_{-2}(\stackrel{*}{x}, \xi, \tau)\right)=\frac{n}{\left(\tau-\mu \sum_{l, m=1}^{n}\left(g^{l m}(\stackrel{*}{x})\right) \xi_{l} \xi_{m}\right)}+\frac{(\mu+\lambda) \sum_{l, m=1}^{n}\left(g^{l m}(\stackrel{*}{x})\right) \xi_{l} \xi_{m}}{\left(\tau-\mu \sum_{l, m=1}^{n}\left(g^{l m}(\stackrel{*}{x})\right) \xi_{l} \xi_{m}\right)\left(\tau-(2 \mu+\lambda) \sum_{l, m=1}^{n}\left(g^{l m}(\stackrel{*}{x})\right) \xi_{l} \xi_{m}\right)} .
$$

This implies that all expressions (4.23)-(4.27) of the above trace symbols have the same form either in $\Omega$ or in $\Omega^{*}$.

For given (small) $\epsilon>0$, denote by $U_{\epsilon}(\partial \Omega)=\{z \in \mathcal{M} \mid \operatorname{dist}(z, \partial \Omega)<\epsilon\}$ the $\epsilon$-neighborhood of $\partial \Omega$ in $\mathcal{M}$. When $x \in \Omega \backslash U_{\epsilon}(\partial \Omega)$, we see by taking geodesic normal coordinate system at $x$ that (4.24) still holds at this $x$. According to (4.25) we have that

$$
\begin{aligned}
\operatorname{Tr}\left(\mathbf{q}_{-2}(t, x, \stackrel{*}{x})\right) & =\frac{1}{(2 \pi)^{n}} \int_{\mathbb{R}^{n}} e^{i\left(x-\frac{*}{x}\right) \cdot \xi}\left((n-1) e^{-t \mu|\xi|^{2}}+e^{-t(2 \mu+\lambda)|\xi|^{2}}\right) d \xi \\
& =\frac{n-1}{(4 \pi \mu t)^{n / 2}} e^{-\frac{\left|x-\frac{*}{2}\right|^{2}}{4 t \mu}}+\frac{1}{(4 \pi(2 \mu+\lambda) t)^{n / 2}} e^{-\frac{\left|x-\frac{*}{x}\right|^{2}}{4 t(2 \mu+\lambda)}} \quad \text { for any } x \in \Omega \backslash U_{\epsilon}(\partial \Omega),
\end{aligned}
$$

which exponentially tends to zero as $t \rightarrow 0^{+}$because $|x-\stackrel{*}{x}| \geq \epsilon$. Hence

$$
\int_{\Omega \backslash U_{\epsilon}(\partial \Omega)}\left(\operatorname{Tr}\left(\mathbf{q}_{-2}(t, x, \stackrel{*}{x})\right)\right) d V=O\left(t^{1-\frac{n}{2}}\right) \quad \text { as } t \rightarrow 0^{+} .
$$

Secondly, for $l \geq 1$, it can be verified that $\operatorname{Tr}\left(\mathbf{q}_{-2-l}(x, \xi, \tau)\right)$ is a sum of finitely many terms, each of which has the following form:

$$
\frac{r_{k}(x, \xi)}{\left(\tau-\mu \sum_{l, m=1}^{n} g^{l m} \xi_{l} \xi_{m}\right)^{s}\left(\tau-(2 \mu+\lambda) \sum_{l, m=1}^{n} g^{l m} \xi_{l} \xi_{m}\right)^{j}},
$$

where $k-2 s-2 j=-2-l$, and $r_{k}(x, \xi)$ is the symbol independent of $\tau$ and homogeneous of degree $k$. Again we take the geodesic normal coordinate systems center at $x$ (i.e., $g_{j k}(x)=\delta_{j k}$ and $\Gamma_{j k}^{l}(x)=0$ ), by applying residue theorem we see that, for $l \geq 1$,

and

$$
\frac{1}{(2 \pi)^{n}} \int_{\mathbb{R}^{n}}\left(\frac{1}{2 \pi i} \int_{\mathcal{C}} e^{-t \tau} \operatorname{Tr}\left(\mathbf{q}_{-2-l}(x, \xi, \tau)\right) d \tau\right) d \xi=O\left(t^{l-\frac{n}{2}}\right) \text { as } t \rightarrow 0^{+} \text {uniformly for } x \in \Omega,
$$

$$
\frac{1}{(2 \pi)^{n}} \int_{\mathbb{R}^{n}} e^{i\left(x-\frac{*}{x}\right) \cdot \xi}\left(\frac{1}{2 \pi i} \int_{\mathcal{C}} e^{-t \tau} \operatorname{Tr}\left(\mathbf{q}_{-2-l}(x, \xi, \tau)\right) d \tau\right) d \xi=O\left(t^{l-\frac{n}{2}}\right) \text { as } t \rightarrow 0^{+} \text {uniformly for } x \in \Omega .
$$

Therefore

$$
\int_{\Omega}\left\{\frac{1}{(2 \pi)^{n}} \int_{\mathbb{R}^{n}}\left(\frac{1}{2 \pi i} \int_{\mathcal{C}} e^{-t \tau} \sum_{l \geq 1} \operatorname{Tr}\left(\mathbf{q}_{-2-l}(x, \xi, \tau)\right) d \tau\right) d \xi\right\} d V=O\left(t^{1-\frac{n}{2}}\right) \text { as } t \rightarrow 0^{+}
$$

and

$$
\int_{\Omega}\left\{\frac{1}{(2 \pi)^{n}} \int_{\mathbb{R}^{n}} e^{i\left(x-\frac{*}{x}\right) \cdot \xi}\left(\frac{1}{2 \pi i} \int_{\mathcal{C}} e^{-t \tau} \sum_{l \geq 1} \operatorname{Tr}\left(\mathbf{q}_{-2-l}(x, \xi, \tau)\right) d \tau\right) d \xi\right\} d V=O\left(t^{1-\frac{n}{2}}\right) \text { as } t \rightarrow 0^{+}
$$

Combining (4.20), (4.27) and (4.30), we have

$$
\int_{\Omega} \operatorname{Tr}(\mathbf{K}(t, x, x)) d V=\left[\frac{n-1}{(4 \pi \mu t)^{n / 2}}+\frac{1}{(4 \pi(2 \mu+\lambda) t)^{n / 2}}\right] \operatorname{Vol}(\Omega)+O\left(t^{1-\frac{n}{2}}\right) \text { as } t \rightarrow 0^{+} .
$$

Finally, we will consider the case of $\int_{\Omega \cap U_{\epsilon}(\partial \Omega)}\left\{\frac{1}{(2 \pi)^{n}} \int_{\mathbb{R}^{n}} e^{i(x-x) \cdot \xi}\left(\frac{1}{2 \pi i} \int_{\mathcal{C}} e^{-t \tau} \operatorname{Tr}\left(\mathbf{q}_{-2}(x, \xi, \tau)\right) d \tau\right) d \xi\right\} d V$. We pick a self-double patch $W$ of $\mathcal{M}$ (such that $W \subset U_{\epsilon}(\partial \Omega)$ ) covering a patch $W \cap \partial \Omega$ of $\partial \Omega$ endowed (see the diagram on p. 54 of [60]) with local coordinates $x$ such that $\epsilon>x_{n}>0$ in $W \cap \Omega ; x_{n}=0$ on $W \cap \partial \Omega$; 


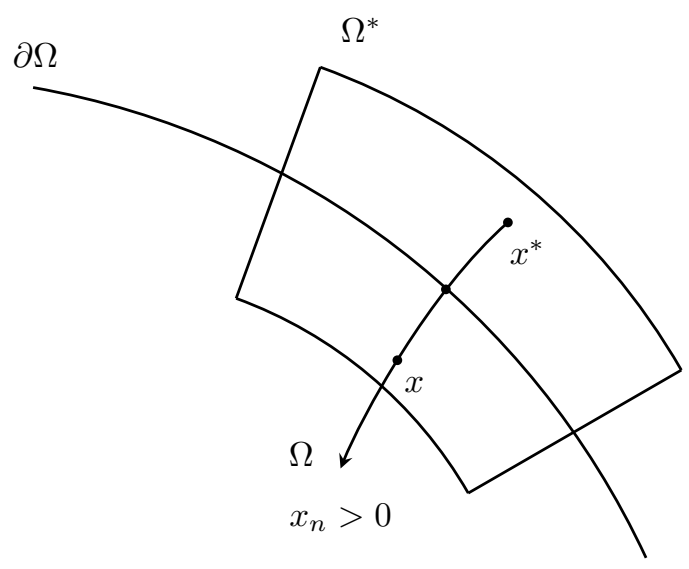

$x_{n}(\stackrel{*}{x})=-x_{n}(x)$; and the positive $x_{n}$-direction is perpendicular to $\partial \Omega$. This has the effect that (4.6)-(4.8) and

$$
\sqrt{|g| / g_{n n}} d x_{1} \cdots d x_{n-1}=\text { the element of (Riemannian) surface area on } \partial \Omega \text {. }
$$

We choose coordinates $x^{\prime}=\left(x_{1}, \cdots, x_{n-1}\right)$ on an open set in $\partial \Omega$ and then coordinates $\left(x^{\prime}, x_{n}\right)$ on a neighborhood in $\bar{\Omega}$ such that $x_{n}=0$ on $\partial \Omega$ and $\left|\nabla x_{n}\right|=1$ near $\partial \Omega$ while $x_{n}>0$ on $\Omega$ and such that $x^{\prime}$ is constant on each geodesic segment in $\bar{\Omega}$ normal to $\partial \Omega$. Then the metric tensor on $\bar{\Omega}$ has the form (see [49] or p. 532 of $[75$ )

$$
\left(g_{j k}\left(x^{\prime}, x_{n}\right)\right)_{n \times n}=\left(\begin{array}{cc}
\left(g_{j k}\left(x^{\prime}, x_{n}\right)\right)_{(n-1) \times(n-1)} & 0 \\
0 & 1
\end{array}\right) .
$$

Furthermore, we can take a geodesic normal coordinate system for $(\partial \Omega, g)$ centered at $x_{0}=0$, with respect to $e_{1}, \cdots, e_{n-1}$, where $e_{1}, \cdots, e_{n-1}$ are the principal curvature vectors. As Riemann showed, one has (see p. 555 of [75])

$$
\begin{gathered}
g_{j k}\left(x_{0}\right)=\delta_{j k}, \quad \frac{\partial g_{j k}}{\partial x_{l}}\left(x_{0}\right)=0 \text { for all } 1 \leq j, k, l \leq n-1, \\
-\frac{1}{2} \frac{\partial g_{j k}}{\partial x_{n}}\left(x_{0}\right)=\kappa_{k} \delta_{j k} \text { for all } 1 \leq j, k \leq n-1,
\end{gathered}
$$

where $\kappa_{1} \cdots, \kappa_{n-1}$ are the principal curvatures of $\partial \Omega$ at point $x_{0}=0$. Due to the special geometric normal coordinate system and (4.35)-(4.34), we see that for any $x \in\{z \in \Omega \mid \operatorname{dist}(z, \partial \Omega)<\epsilon\}$,

$$
x-\stackrel{*}{x}=\left(0, \cdots, 0, x_{n}-\left(-x_{n}\right)\right)=\left(0, \cdots, 0,2 x_{n}\right) .
$$

By (3.18), (4.35), (4.25) and (4.36), we find that

$$
\begin{aligned}
& \int_{W \cap \Omega}\left\{\frac{1}{(2 \pi)^{n}} \int_{\mathbb{R}^{n}} e^{i\langle x-* x, \xi\rangle}\left(\frac{1}{2 \pi i} \int_{\mathcal{C}} e^{-t \tau} \operatorname{Tr}\left(\mathbf{q}_{-2}(x, \xi, \tau)\right) d \tau\right) d \xi\right\} d V \\
& =\int_{0}^{\epsilon} d x_{n} \int_{W \cap \partial \Omega} \frac{d x^{\prime}}{(2 \pi)^{n}} \int_{\mathbb{R}^{n}} e^{i\left\langle 0, \xi^{\prime}\right\rangle+i 2 x_{n} \xi_{n}}\left[\frac{1}{2 \pi i} \int_{\mathcal{C}} e^{-t \tau}\left(\frac{n}{\left(\tau-\mu|\xi|^{2}\right)}+\frac{(\mu+\lambda)|\xi|^{2}}{\left(\tau-\mu|\xi|^{2}\right)\left(\tau-(2 \mu+\lambda)|\xi|^{2}\right)}\right) d \tau\right] d \xi \\
& =\int_{0}^{\epsilon} d x_{n} \int_{W \cap \partial \Omega} \frac{d x^{\prime}}{(2 \pi)^{n}} \int_{\mathbb{R}^{n}} e^{i 2 x_{n} \xi_{n}}\left((n-1) e^{-t \mu|\xi|^{2}}+e^{-t(2 \mu+\lambda)|\xi|^{2}} d \tau\right) d \xi \\
& =\int_{0}^{\epsilon} d x_{n} \int_{W \cap \partial \Omega} \frac{d x^{\prime}}{(2 \pi)^{n}} \int_{-\infty}^{\infty} e^{2 i x_{n} \xi_{n}}\left[\int_{\mathbb{R}^{n-1}}\left((n-1) e^{-t \mu\left(\left|\xi^{\prime}\right|^{2}+\xi_{n}^{2}\right)}+e^{-t(2 \mu+\lambda)\left(\left|\xi^{\prime}\right|^{2}+\xi_{n}^{2}\right)}\right) d \xi^{\prime}\right] d \xi_{n} \\
& =\int_{0}^{\epsilon} d x_{n} \int_{W \cap \partial \Omega} \frac{1}{(2 \pi)^{n}}\left[\int_{-\infty}^{\infty} e^{2 i x_{n} \xi_{n}} e^{-t \mu \xi_{n}^{2}}\left(\int_{\mathbb{R}^{n-1}}(n-1) e^{-t \mu \sum_{j=1}^{n-1} \xi_{j}^{2}} d \xi^{\prime}\right) d \xi_{n}\right] d x^{\prime} \\
& \quad+\int_{0}^{\epsilon} d x_{n} \int_{W \cap \partial \Omega} \frac{1}{(2 \pi)^{n}}\left[\int_{-\infty}^{\infty} e^{2 i x_{n} \xi_{n}} e^{-t(2 \mu+\lambda) \xi_{n}^{2}}\left(\int_{\mathbb{R}^{n-1}} e^{-t(2 \mu+\lambda) \sum_{j=1}^{n-1} \xi_{j}^{2}} d \xi^{\prime}\right) d \xi_{n}\right] d x^{\prime},
\end{aligned}
$$


where $\xi=\left(\xi^{\prime}, \xi_{n}\right) \in \mathbb{R}^{n}, \xi^{\prime}=\left(\xi_{1}, \cdots, \xi_{n-1}\right)$. A direct calculation shows that

$$
\begin{aligned}
& \frac{1}{(2 \pi)^{n}}\left[\int_{-\infty}^{\infty} e^{2 i x_{n} \xi_{n}} e^{-t \mu \xi_{n}^{2}}\left(\int_{\mathbb{R}^{n-1}}(n-1) e^{-t \mu \sum_{j=1}^{n-1} \xi_{j}^{2}} d \xi^{\prime}\right) d \xi_{n}\right]=\frac{n-1}{(4 \pi \mu t)^{n / 2}} e^{-\frac{\left(2 x_{n}\right)^{2}}{4 \mu t}} \\
& \frac{1}{(2 \pi)^{n}}\left[\int_{-\infty}^{\infty} e^{2 i x_{n} \xi_{n}} e^{-t(2 \mu+\lambda) \xi_{n}^{2}}\left(\int_{\mathbb{R}^{n-1}} e^{-t(2 \mu+\lambda) \sum_{j=1}^{n-1} \xi_{j}^{2}} d \xi^{\prime}\right) d \xi_{n}=\frac{1}{(4 \pi(2 \mu+\lambda) t)^{n / 2}} e^{-\frac{\left(2 x_{n}\right)^{2}}{4(2 \mu+\lambda) t}} .\right.
\end{aligned}
$$

Hence

$$
\begin{aligned}
\int_{W \cap \Omega} & \left\{\frac{1}{(2 \pi)^{n}} \int_{\mathbb{R}^{n}} e^{i\left\langle x-x^{*}, \xi\right\rangle}\left(\frac{1}{2 \pi i} \int_{\mathcal{C}} e^{-t \tau} \operatorname{Tr}\left(\mathbf{q}_{-2}(x, \xi, \tau)\right) d \tau\right) d \xi\right\} d V \\
= & \int_{0}^{\epsilon} d x_{n} \int_{W \cap \partial \Omega}\left[\frac{n-1}{(4 \pi \mu t)^{n / 2}} e^{-\frac{\left(2 x_{n}\right)^{2}}{4 \mu t}}+\frac{1}{(4 \pi(2 \mu+\lambda) t)^{n / 2}} e^{-\frac{\left(2 x_{n}\right)^{2}}{4(2 \mu+\lambda) t}}\right] d x^{\prime} \\
= & \int_{0}^{\infty} d x_{n} \int_{W \cap \partial \Omega}\left[\frac{n-1}{(4 \pi \mu t)^{n / 2}} e^{-\frac{\left(2 x_{n}\right)^{2}}{4 \mu t}}+\frac{1}{(4 \pi(2 \mu+\lambda) t)^{n / 2}} e^{-\frac{\left(2 x_{n}\right)^{2}}{4(2 \mu+\lambda) t}}\right] d x^{\prime} \\
& -\int_{\epsilon}^{\infty} d x_{n} \int_{W \cap \partial \Omega}\left[\frac{n-1}{(4 \pi \mu t)^{n / 2}} e^{-\frac{\left(2 x_{n}\right)^{2}}{4 \mu t}}+\frac{1}{(4 \pi(2 \mu+\lambda) t)^{n / 2}} e^{-\frac{\left(2 x_{n}\right)^{2}}{4(2 \mu+\lambda) t}}\right] d x^{\prime} \\
= & \frac{n-1}{4} \cdot \frac{\operatorname{Vol}(W \cap \partial \Omega)}{(4 \pi \mu t)^{(n-1) / 2}+\frac{1}{4} \cdot \frac{\operatorname{Vol}(W \cap \partial \Omega)}{(4 \pi(2 \mu+\lambda) t)^{(n-1) / 2}}} \\
& -\int_{W \cap \partial \Omega}\left\{\int_{\epsilon}^{\infty}\left[\frac{n-1}{(4 \pi \mu t)^{n / 2}} e^{-\frac{\left(2 x_{n}\right)^{2}}{\mu \mu t}}+\frac{1}{(4 \pi(2 \mu+\lambda) t)^{n / 2}} e^{-\frac{\left(2 x_{n}\right)^{2}}{4(2 \mu+\lambda) t}}\right] d x_{n}\right\} d x^{\prime} .
\end{aligned}
$$

It is easy to verify that for any fixed $\epsilon>0$,

$$
\begin{aligned}
& \int_{\epsilon}^{\infty} \frac{1}{(4 \pi \lambda t)^{\frac{n}{2}}} e^{-\frac{\left(2 x_{n}\right)^{2}}{4 \mu t}} d x_{n}=O\left(t^{1-n / 2}\right) \quad \text { as } t \rightarrow 0^{+}, \\
& \int_{\epsilon}^{\infty} \frac{1}{(4 \pi(2 \mu+\lambda) t)^{\frac{n}{2}}} e^{-\frac{\left(2 x_{n}\right)^{2}}{4(2 \mu+\lambda) t}} d x_{n}=O\left(t^{1-n / 2}\right) \quad \text { as } t \rightarrow 0^{+} .
\end{aligned}
$$

From (4.37) and (4.38), we get that

$$
\begin{aligned}
\int_{W \cap \Omega}\{ & \left.\frac{1}{(2 \pi)^{n}} \int_{\mathbb{R}^{n}} e^{i\langle x-* * * \xi\rangle}\left(\frac{1}{2 \pi i} \int_{\mathcal{C}} e^{-t \tau} \operatorname{Tr}\left(\mathbf{q}_{-2}(x, \xi, \tau)\right) d \tau\right) d \xi\right\} d V=\frac{n-1}{4} \cdot \frac{\operatorname{Vol}(W \cap \partial \Omega)}{(4 \pi \mu t)^{(n-1) / 2}} \\
& +\frac{1}{4} \cdot \frac{\operatorname{Vol}(W \cap \partial \Omega)}{(4 \pi(2 \mu+\lambda) t)^{(n-1) / 2}}+O\left(t^{1-n / 2}\right) \quad \text { as } t \rightarrow 0^{+} .
\end{aligned}
$$

For any $x \in \Omega \cap U_{\epsilon}(\partial \Omega)$, we have

$$
\begin{aligned}
\operatorname{Tr}(K(t, x, \stackrel{*}{x}))= & \frac{1}{(2 \pi)^{n}} \int_{\mathbb{R}^{n}} e^{i\langle x-\stackrel{*}{x}, \xi\rangle}\left(\frac{1}{2 \pi i} \int_{\mathcal{C}} e^{-t \tau} \operatorname{Tr}\left(\mathbf{q}_{-2}(x, \xi, \tau)\right) d \tau\right) d \xi \\
& +\frac{1}{(2 \pi)^{n}} \int_{\mathbb{R}^{n}} e^{i\langle x-x, \xi\rangle}\left(\sum_{l \geq 1} \frac{1}{2 \pi i} \int_{\mathcal{C}} e^{-t \tau} \operatorname{Tr}\left(\mathbf{q}_{-2-l}(x, \xi, \tau)\right) d \tau\right) d \xi \\
= & \frac{1}{(2 \pi)^{n}} \int_{\mathbb{R}^{n}} e^{i\langle x-\stackrel{*}{x}, \xi\rangle}\left(\frac{1}{2 \pi i} \int_{\mathcal{C}} e^{-t \tau} \operatorname{Tr}\left(\mathbf{q}_{-2}(x, \xi, \tau)\right) d \tau\right) d \xi+O\left(t^{1+\frac{n}{2}}\right) \text { as } t \rightarrow 0^{+},
\end{aligned}
$$

where the second equality used (4.29). Combining (4.39) and (4.40), we have

$$
\begin{aligned}
\int_{W \cap \Omega} \operatorname{Tr} & (\mathbf{K}(t, x, \stackrel{*}{x})) d x=\frac{n-1}{4} \cdot \frac{\operatorname{Vol}(W \cap \partial \Omega)}{(4 \pi \mu t)^{(n-1) / 2}} \\
& +\frac{1}{4} \cdot \frac{\operatorname{Vol}(W \cap \partial \Omega)}{(4 \pi(2 \mu+\lambda) t)^{(n-1) / 2}}+O\left(t^{1-n / 2}\right) \quad \text { as } t \rightarrow 0^{+} .
\end{aligned}
$$

It follows from (4.15), (4.21), (4.28), (4.31), (4.32) and (4.41) that

$$
\int_{W \cap \Omega} \operatorname{Tr}\left(\mathbf{K}^{\mp}(t, x, x)\right) d x=\int_{W \cap \Omega} \operatorname{Tr}(\mathbf{K}(t, x, x)) d x \mp \int_{W \cap \Omega} \operatorname{Tr}(\mathbf{K}(t, x, \stackrel{*}{x})) d x
$$




$$
\begin{aligned}
= & {\left[\frac{n-1}{(4 \pi \mu t)^{n / 2}}+\frac{1}{(4 \pi(2 \mu+\lambda) t)^{n / 2}}\right] \operatorname{Vol}(W \cap \Omega) } \\
& \mp \frac{1}{4}\left[(n-1) \frac{\operatorname{Vol}(W \cap \partial \Omega)}{(4 \pi \mu t)^{(n-1) / 2}}+\frac{\operatorname{Vol}(W \cap \partial \Omega)}{(4 \pi(2 \mu+\lambda) t)^{(n-1) / 2}}\right] \\
& +O\left(t^{1-n / 2}\right) \quad \text { as } t \rightarrow 0^{+},
\end{aligned}
$$

and hence (1.11) holds.

Remark 4.1. i) It is very clear that our method and results are still valid in the case of Euclidean space. In other words, if $\Omega$ is a bounded domain in $\mathbb{R}^{n}$, and if $\left\{\tau_{k}^{-}\right\}$and $\left\{\tau_{k}^{+}\right\}$respectively be all the Navier-Lamé eigenvalues corresponding to the Navier-Lamé operator $P \mathbf{u}=-\mu \Delta \mathbf{u}-(\mu+\lambda) \nabla(\nabla \cdot \mathbf{u})$, with the Dirichlet and Neumann boundary condition, then the asymptotic formula (1.11) still holds, where the $\operatorname{Vol}(\Omega)$ and $\operatorname{Vol}(\partial \Omega)$ are to be replaced by the $n$-dimensional Euclidean volume $|\Omega|$ and $(n-1)$-dimensional Euclidean volume $|\partial \Omega|$, respectively.

ii) Note that (see [5, [10, [11, 28, 61] or [32])

$$
\int_{\Omega} \operatorname{Tr}\left(\mathbf{K}^{\mp}(t, x, x)\right) d x=(4 \pi t)^{-n / 2}\left[a_{0}+a_{1}^{\mp} t^{1 / 2}+a_{2}^{\mp} t+\cdots+a_{m}^{\mp} t^{m / 2}+O\left(t^{(m+1) / 2}\right)\right] \text { as } t \rightarrow 0^{+} .
$$

Except for the above obtained $a_{0}$ and $a_{1}^{\mp}$, we can also get all coefficients $a_{l}^{\mp}, 2 \leq l \leq m$, for the asymptotic expansion of the integral of trace of integral kernel for the Navier-Lamé operator by our new method.

Now, we use the geometric invariants of the Navier-Lamé spectrum which have been obtained from Theorem 1.1 to finish the proof of Theorem 1.2.

Proof of Theorem 1.2. By Theorem 1.1, we know that the first two coefficients $a_{0}$ and $a_{1}$ of the asymptotic expansion in (1.11) are Navier-Lamé spectral invariants. From the expressions of $a_{0}$ and $a_{1}$, we can further know that $|\Omega|=\left|B_{r}\right|$ and $|\partial \Omega|=\left|\partial B_{r}\right|$. That is, $\frac{|\partial \Omega|}{|\Omega|^{(n-1) / n}}=\frac{\left|\partial B_{r}\right|}{\left|B_{r}\right|^{(n-1) / n}}$. Note that for any $r>0, \frac{\left|\partial B_{r}\right|}{\left|B_{r}\right|^{(n-1) / n}}=$

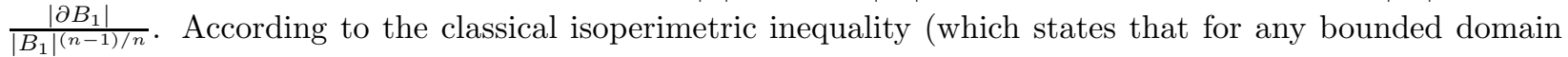
$\Omega \subset \mathbb{R}^{n}$ with smooth boundary, the following inequality holds:

$$
\frac{|\partial \Omega|}{|\Omega|^{(n-1) / n}} \geq \frac{\left|\partial B_{1}\right|}{\left|B_{1}\right|^{(n-1) / n}}
$$

Moreover, equality obtains if and only if $\Omega$ is a ball, see [17] or p. 183 of [18]), we immediately get $\Omega=B_{r}$.

Remark 4.2. $\quad$ By applying the Tauberian theorem (see, for example, Theorem 15.3 of p. 30 of [45] or p. 446 of [24]) for the first term on the right side of (1.11) (i.e., $\sum_{k=1}^{\infty} e^{-t \tau_{k}^{\mp}}=\int_{0}^{\infty} e^{-t \eta} d N^{\mp}(\eta)=\left[\frac{n-1}{(4 \pi \mu t)^{n / 2}}+\right.$ $\left.\frac{1}{(4 \pi(2 \mu+\lambda) t)^{n / 2}}\right] \operatorname{Vol}(\Omega)+o\left(t^{-n / 2}\right)$ as $\left.t \rightarrow 0^{+}\right)$, we can easily obtain the Weyl-type law for the Navier-Lamé eigenvalues:

$$
\begin{aligned}
N^{\mp}(\eta) & =\max \left\{k \mid \tau_{k}^{\mp} \leq \eta\right\} \\
& =\frac{\operatorname{Vol}(\Omega)}{\Gamma\left(\frac{n}{2}+1\right)}\left[\frac{n-1}{(4 \pi \mu)^{n / 2}}+\frac{1}{(4 \pi(2 \mu+\lambda))^{n / 2}}\right] \eta^{\frac{n}{2}}+o\left(\eta^{\frac{n}{2}}\right), \quad \text { as } \eta \rightarrow+\infty .
\end{aligned}
$$

Remark 4.3. Note that as $\lambda \rightarrow-\mu$, the Navier-Lamé operator reduces to the classical Laplacian. Therefore our results recover all corresponding results for the Laplacian by letting $\lambda+\mu=0$ (cf. [67] and [53]).

Remark 4.4. Besides the elastic Navier-Lamé system, by applying our new technique we can also explicitly calculate and obtain the full symbol of the resolvent operator for the thermoelastic system [58] (it is also an elliptic system). Furthermore, the parabolic trace for this elliptic system can also be explicitly obtained. The thermoelastic system is an important mathematical model, which gives the laws obtained by the deformation of a thermoelastic body and its interior temperature distribution (see Chapter of [50]).

Generally, for any matrix-valued elliptic operator $L$, if one can explicitly obtain the inverse matrix for the principal symbol of the operator $(\tau I-L)^{-1}$, then all coefficients in the asymptotic expansion of the parabolic trace will immediately be obtained by our new (algorithm) method. 


\section{Acknowledgments}

This research was supported by NNSF of China (11671033/A010802) and NNSF of China (11171023/A010801).

\section{REFERENCES}

1. S. Agmon, On kernels, eigenvalues, and eigenfunctions of operators related to elliptic problems, Comm. Pure Appl. Math., No.4, 18(1965), 627-663.

2. L. Ahlfors, Complex Analysis, Third Edition, McGraw-Hill, 1979.

3. E. Artin, Galois theory, Notre Dame Univ., Indiana, 1942.

4. M. S. Ashbaugh, F. Gesztesy, M. Mitrea, G. Teschl, Spectral theory for perturbed Krein Laplacians in nonsmooth domains, Advances in Mathematics, 223(2010), 1372-1467.

5. I. G. Avramidi, Heat kernel approach in quantum field theory, Nucl. Phys. Proc. Suppl. 104 (2002), 3-32.

6. I. G. Avramidi, A method for calculating the heat kernel for manifolds with boundary, Yadernaya Fizika, 56 (1993) $245-252$ [Russian]; Physics of Atomic Nucleus, 56 (1993), 138-142 [English].

7. I. G. Avramidi, Matrix general relativity: a new look at old problems, Class. Quant. Grav., 21 (2004) 103-120.

8. I. G. Avramidi, Gauged gravity via spectral asymptotics of non-Laplace type operators, J. High Energy Phys., 07 (2004), 030.

9. I. G. Avramidi, Dirac operator in matrix geometry, Int. J. Geom. Methods Mod. Phys., 2 (2005), 227-264.

10. I. G. Avramidi, Non-Laplace type operators on manifolds with boundary, Analysis, geometry and topology of elliptic operators, World Sci. Publ., Hackensack, NJ (2006), 107-140.

11. I. G. Avramidi, Heat Kernel and Quantum Gravity, Lecture Notes in Physics, New Series m: Monographs, 64, SpringerVerlag, Berlin, 2000.

12. A. Bantle, Effecient implementation of the collocation method for the Navier-Lamé equation, June 2010.

13. N. Berline, E. Getzler and M. Vergne, Heat Kernels and Dirac Operators, Berlin, Springer-Verlag, 1992.

14. T. P. Branson and P. B. Gilkey, The asymptotics of the Laplacian on a manifold with boundary, Comm. Partial Differential Equations, 15(1990), 245-272.

15. T. Branson, P. Gilkey, K. Kirsten and D. Vassilevich, Heat kernel asymptotics with mixed boundary conditions, Nucl. Phys. B563 (1999), 603-626.

16. F. E. Browder, On the spectral theory of elliptic differential operators I, Math. Ann. 142(1960/61), 22-130.

17. I. Chavel, Eigenvalues in Riemannian Geometry, Academic Press, 1984.

18. B. Chow, P. Lu and L. Ni, Hamilton's Ricci flow, Science Press, Beijing, American Mathematical Society, Providence, RI, 2006.

19. D. M. Cook, The Theory of the Electromagnetic Field, Mineola NY: Courier Dover Publications, 2002.

20. H. Dong, Gradient Estimates for Parabolic and Elliptic Systems from Linear Laminates. Arch Rational Mech Anal 205, 119-149 (2012). DOI: 10.1007/s00205-012-0501-z.

21. B. A. Dubrovin, A. T. Domenko, S. P. Novikov, Modern Geometry-Methods and Applications, Part I. The geometry of surfaces, transformation groups, and Fields, Springer-Verlag, New York Inc., 1984.

22. H. M. Edwards, Galois theory, Springer-Verlag, 1984.

23. L. C. Evans, Partial Differential Equations, American Mathematical Society, Second Edition, 2010.

24. W. Feller, An Introduction to Probability Theory and its Applications, Vol.I, Third Edition, John Wiley \& Sons, Inc., New York-London-Sydney, 1968.

25. A. Friedman, Partial Differential Equations of Parabolic Type, Prentice Hall, Englewood Cliffs, NJ, 1964.

26. P. Gilkey, Recursion relations and the asymptotic behavior of the eigenvalues of the Laplacian, Compositio Math., 38(1979), 201-240.

27. P. Gilkey, The spectral geometry of a Riemannian manifold, J. Differential Geometry, 10(1975), 601-618.

28. P. Gilkey, Invariance Theory, the Heat Equation and the Atiyah-Singer Index Theorem, CRC Press, Boca Raton, 1995.

29. H. Gimperlein and G. Grubb, Heat kernel estimates for pseudodifferential operators, fractional Laplacians and Dirichletto-Neumann operators, J. Evolution Eq., 14 (2014), 49-83.

30. P. Greiner, An asymptotic expansion for the heat equation, Arch. Rational Mech. Anal., 41(1971), 163-218.

31. D. Griffiths, Introduction to electrodynamics (Third ed.), Prentice Hall. pp. 559-562, 1999.

32. G. Grubb, Functional Calculus of Pseudo-differential Boundary Problems, Birkhäuser, Boston, 1986.

33. G. Grubb, Distributions and Operators, Graduate Texts in Mathematics, vol.252, Springer, New York, 2009.

34. M. Gurtin, The Linear Theory of Elasticity, C. Truesdell (Ed.), Handbuch der Physik, VI, Springer-Verlag, New York, 1972.

35. S. M. Hook, Domain independent upper bounds for eigenvalues of elliptic operator, Trans. Amer. Math. Soc. 318(1990), 615-642.

36. L. Hörmander, The Analysis of Partial Differential Operators III, Springer-Verlag, Berlin Heidelberg New York, 1985.

37. L. Hörmander, The Analysis of Partial Differential Operators IV, Springer-Verlag, Berlin Heidelberg New York, 1985.

38. M. Kac, Can one hear the shape of a drum?, Amer. Math. Monthly (Slaught Mem. Papers, no. 11), 73(4)(1966), 1-23. 
39. B. Kawohl, Remarks on some old and current eigenvalue problems, in: Partial Differential Equations of Elliptc Type, Edited by A. Alvino, E. Fabes and G. Talenti, Cambridge University Press, 1994, 165-183.

40. B. Kawohl, H. Levine and W. Velte, Bucklinf eigenvalues for a clamped plate embedded in an elastic medium and related questions, SIAM J. Math. Anal. 24(1993), 327-340.

41. B. Kawohl and G. Sweers, Remarks on eigenvalues and eigenfunctions of a special elliptic system, J. Applied Math. Phys. (ZAMP), 38(1987), 730-740.

42. K. Kirsten, Spectral Functions in Mathematics and Physics, CRC Press, Boca Raton, 2001.

43. K. Kirsten, The $a_{5}$ heat kernel coefficient on a manifold with boundary, Class. Quant. Grav. 15 (1998), L5-L12.

44. J. Kohn and L. Nirenberg, An algebra of pseudo-differential operators. Comm. Pure Appl. Math., 18(1965), 269-305.

45. J. Korevaar, Tauberian Theory: A Century of Developments, Springer-Verlag, Berlin, Heidelberg, 2004.

46. L. Landau and E. M. Lifshitz, Theory of Elasticity (3rd ed.), Oxford, England: Butterworth Heinemann, 1986.

47. A. Laptev, Dirichlet and Neumann Eigenvalue Problems on Domainsin Euclidean Spaces, Journal of Functional Analysis, 151(1997), 531-545.

48. J. M. Lee, Introduction to smooth manifolds, Second Edition, Springer-Verlag+Business Media New York, 2013.

49. J. Lee and G. Uhlmann, Determing anisotropic real-analytic conductivities by boundary measurements, Comm. Pure Appl. Math., 42(1989), 1097-1112.

50. T. Li and T. Qin, Physics and partial differential equations, vol. 2, SIAM \& Higher Education Press, 2014.

51. G. Q. Liu, The Weyl-type asymptotic formula for biharmonic Steklov eigenvalues on Riemannian manifolds, Advances in Math., 228 (2011), 2162-2217.

52. G. Q. Liu, The geometric invariants for the spectrum of the Stokes operator, Math. Ann., 2022, 382(3-4), 1985-2032. (DOI: 10.1007/s00208-021-02167-w)

53. G. Q. Liu, Asymptotic expansion of the trace of the heat kernel associated to the Dirichlet-to-Neumann operator, J. Differential Equations, 259(2015), 2499-2545.

54. G. Q. Liu, Spectral invariants of the perturbed polyharmonic Steklov problem, Calc. Var. Partial Differential Equations, (2022) 61:125.

55. G. Q. Liu, Geometric Invariants of Spectrum of the Navier-Lamé Operator, The Journal of Geometric Analysis, 2021, 31(10): 10164-10193.(DOI: 10.1007/s12220-021-00639-8).

56. G. Q. Liu, Determination of isometric real-analytic metric and spectral invariants for elastic Dirichlet-to-Neumann map on Riemannian manifolds, arXiv: 1908.05096v2 [math.AP].

57. G. Q. Liu, Determining anisotropic real-analytic metric from boundary electromagnetic information, arXiv: 1909.12803v2 [math.AP].

58. G. Q. Liu, X. Tan, Asymptotic Expansions of the Traces of the Thermoelastic Operators, arXiv: 2205.13238 [math.SP].

59. G. G. Lorentz, Beweis des Gausschen Intergralsatzes, Math. Z., 51(1949), 61-81.

60. H. McKean and I. M. Singer, Curvature and the eigenvalues of the Laplacian, J. Differential Geometry, 1(1967), $43-69$.

61. S. Minakshisundaram, Eigenfunctions on Riemanniun manifolds, J. Indian Math. Soc. 17(1953) 158-165.

62. C. Morrey, On the analyticity of the solutions of analytic non-linear elliptic systems of partial differential equations, Amer. J. Math. 80(1958), 198-218.

63. C. Morrey, On the analyticity of the solutions of analytic non-linear elliptic systems of partial differential equations, Amer. J. Math. 80(1958), 219-237.

64. C. Morrey, Multiple Integrals in the Calculus of Variations, Springer-Verlag, New York, Inc., 1966.

65. A. Pazy, Semigroups of Linear Operators and Applications to Partial Differential Equations (Springer, 1983).

66. A. Pleijel, Proprietés asymptotique des fonctions fondamentales du problems des vibrations dans un corps élastique, Ark. Mat. Astron. Fys. 26(1939), 1-9.

67. I. Polterovich and D. Sher, Heat invariants of the Steklov problem, J. Geom. Analysis 25(2015), no.2, 924-950.

68. R. Seeley, Complex powers of an elliptic operator, Proceedings of Symposia in Pure Mathematics, 10(1967), 288-307.

69. R. Seeley, The resolvent of an elliptic boundary value problem, Am. J. Math., 91(1969), 889-920.

70. W. S. Slaughter, The linearized theory of elasticity, Birkhauser, 2002.

71. A. Sommerfeld, Mechanics of Deformable Bodies, New York: Academic Press, 1964.

72. H. B. Stewart, Generation of analytic semigroups by strongly elliptic operators, Trans. Amer. Math. Soc., 199(1974) 141-161.

73. J. A. Stratton, Electromagnetic Theory. McGraw-Hill Book Company, 1941.

74. M. E. Taylor, Partial Differential Equations I, 2nd Edition, Appl. Math. Sci., vol. 115, Springer Science+Business Media, LLC 1996, 2011.

75. M. E. Taylor, Partial Differential Equations II, 2nd Edition, Appl. Math. Sci., vol. 116, Springer Science+Business Media, LLC 1996, 2011.

76. D. Vassilevich, Heat kernel expansion: user's manual, Phys. Rept. 388 (2003), 279-360.

77. H. Weyl, Über die Abhängigkeit der Eigenschwingungen einer Membran und deren Begrenzung, J. Reine Angew. Math., 141(1912), 1-11.

78. J. G. Xiong, $C^{1,1}$ Estimates for elliptic equations with partial and piecewise continuous coefficients, Methods and Applications of Analysis, No, 4, 18(2011), 373-390.

School of Mathematics and Statistics, Beijing Institute of Technology, Beijing 100081, China

Email address: liugqz@bit.edu.cn 